\title{
Wake-function, impedance, and energy loss determination for two countermoving particle beams
}

\author{
L. Teofiliø, ${ }^{1, *}$ P. Arpaia $\odot,{ }^{2, *}$ F. Giordano $\odot,{ }^{2, *}$ I. Lamas $\odot,{ }^{3}$ G. Rumolo, ${ }^{3}$ B. Salvant, ${ }^{3}$ \\ C. Zannini ${ }^{3}{ }^{3}$ and M. Migliorati ${ }^{1, \dagger}$ \\ ${ }^{1}$ University of Rome La Sapienza and INFN Sezione Romal, 00185 Rome, Italy \\ ${ }^{2}$ University of Naples Federico II, 80138 Naples, Italy \\ ${ }^{3}$ CERN, 1217 Geneva, Switzerland
}

(Received 6 July 2020; accepted 25 February 2021; published 1 April 2021)

\begin{abstract}
This paper deals with the determination of the wake-function, impedance and energy loss due to surrounding accelerator components of two countermoving beams, i.e., two beams moving in opposite directions. Differently from the classical case, the distance between source and test charges cannot be considered constant. The paper defines a generalized wake-function, independent of the source and test particle's direction of motion. From this generalized wake-function a wake-potential is derived. The paper focuses in particular on the longitudinal direction. The expression of the energy dissipated by two beams transiting in the same vacuum chamber is obtained. This expression is valid both in the comoving and countermoving beam scenario. Subsequently, the proposed model is benchmarked by calculating the longitudinal co and countermoving wake-functions, the corresponding impedances and wake-potentials for two simple geometries (a circular pipe and a pillbox cavity) traversed by two countermoving beams, and by comparing the results with the ones of the electromagnetic code CST Studio Suite. Finally, for the pillbox geometry, the energy loss of the two countermoving beams and their rf-heating are investigated.
\end{abstract}

DOI: 10.1103/PhysRevAccelBeams.24.041001

\section{INTRODUCTION}

The particles of a beam traversing a vacuum chamber interact electromagnetically with it generating the so-called wakefields. These wakefields dissipate heat on the vacuum chamber materials (rf-heating) and act back on the beam particles potentially triggering instabilities. In the case of a single beam traversing the vacuum chamber all the particles move in the same direction. Using the nomenclature proposed by Wang [1], this paper names this scenario comoving wakefield or simply wakefield (in Fig. 1(a) two comoving particles are reported, most of the quantities used in the paper are also presented).

The physical model for quantifying the effects of the comoving wakefield has been the object of many studies, and the works of Chao [2], $\mathrm{Ng}$ [3], Zotter and Kheifets [4] and Bane et al. [5] are examples of the basis of the current understanding on the topic.

However, in some devices of circular colliders there is an extra complication due to the presence of two

\footnotetext{
*Also at CERN, 1217 Geneva, Switzerland.

mauro.migliorati@uniroma1.it
}

Published by the American Physical Society under the terms of the Creative Commons Attribution 4.0 International license. Further distribution of this work must maintain attribution to the author(s) and the published article's title, journal citation, and DOI. counterrotating particle beams. Usually, for large modern circular colliders, the two beams pass in two separate vacuum chambers. However, in the collision regions and, sometimes in other components, they transit in the same vacuum chamber. In this case, the particles of one beam move in opposite direction with respect to the particles of the other beam. Each beam generates its own wakefield that acts on itself and on the counterrotating beam. This scenario is relevant also in the collision chamber of linear colliders. Once again, using the nomenclature introduced by Wang [1], one refers to the latter case as countermoving wakefields (in Fig. 1(b) two countermoving particles are reported).

In the Large Hadron Collider (LHC) [6] at the laboratories of the European Council for Nuclear Research (CERN) two counterrotating beams circulate. They transit in the same vacuum chamber in the collision chambers, at the four interaction points, and in other components such as the Target Dump Injection or TDI [7] (the LHC injection absorber), and the Target Dump Injection Segmented (TDIS) [8], the upgrade of the TDI to be installed in 2020.

In 2011, the TDI had major issues due to unexpected severe rf-heating [9]. A possible explanation could be linked to the rf-heating resulting from the interaction of the two counterrotating beams. To avoid these issues with the TDIS, CERN allocated resources to investigate the power losses to surrounding components of two countermoving beams. This paper reports the results of this study, showing that comoving 


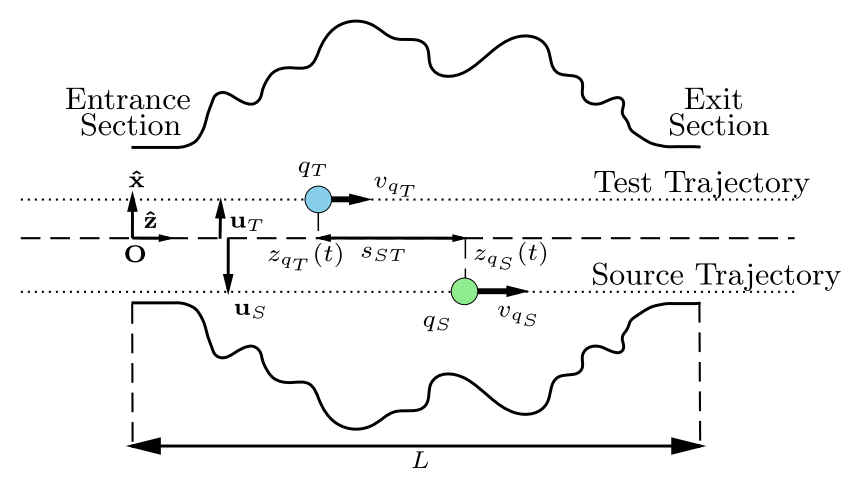

(a)

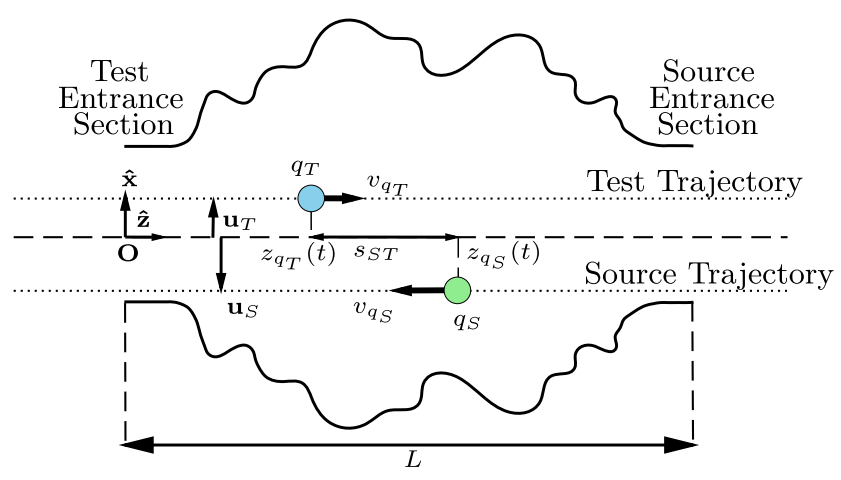

(b)

FIG. 1. Source $\left(q_{S}\right)$ and Test $\left(q_{T}\right)$ charge transiting inside a vacuum chamber. The figure shows the instantaneous longitudinal positions of the two charges $z_{q_{S}}(t)$ and $z_{q_{T}}(t)$, their speeds $v_{q_{S}}$ and $v_{q_{T}}$, their longitudinal distance $s_{S T}(t)$ and the transverse position of their trajectories with respect to the main reference frame $\mathbf{u}_{T}$ and $\mathbf{u}_{S}$. The fixed reference system $\mathbf{O}$ has origin in the entrance section of the test particle and the $\hat{\mathbf{z}}$ axis is aligned with the test particle velocity vector. The length of the vacuum chamber $L$ is also indicated. (a) Comoving case, $\mathrm{S}$ and $\mathrm{T}$ move in the same direction. (b) Countermoving case, $\mathrm{S}$ and T move in opposite direction.

and countermoving beams, transiting into a vacuum chamber, can lead to additional resonance conditions that should be considered to mitigate rf-heating.

In the past, few studies have investigated the interaction between two countermoving beams via their wakefield: Pellegrini [10] and Wang [1] studied longitudinal and transverse countermoving two beam instabilities linked to resonant modes for the Large Electron Positron storage ring (LEP) [11]. They modeled the resonant modes as if they were generated by circular cavities. The latter were thought of as thin lenses, symmetric along their longitudinal axis. Further, their model focuses on the interaction of a beam with the wakefield remaining in the cavity after the other beam has left the cavity. It does not describe what happens when both the countermoving beams are inside the cavity. Zimmerman [12] discussed the transverse resistive wall wakefield problem for two countermoving beams. Zannini et al. $[13,14]$ and Grudiev [15] presented models to compute the rf-heating induced in a vacuum chamber traversed by the countermoving beams, and recently Giordano et al. [16] have presented a benchmark of these models against simulation results.

In this context, this paper focuses on the computation of the countermoving beams induced heating. It is not the aim of the paper to discuss beam instabilities. The paper proposes and benchmark a comprehensive formal physical model to obtain the comoving and countermoving wake-function and wake-potential and, from them, the beam energy loss.

Contrary to the models of Wang [1] and Pellegrini [10], the proposed model can take into account also the wakefield effects generated when both the beams are still inside the vacuum chamber. Regarding the power computation, the proposed model can reobtain results that are similar to the ones of Grudiev and Zannini $[13,15]$. Differently from these works, it is able to take into account also the transient wakefield rf-heating effects that are generated when both beams transit inside the vacuum chamber.
The paper has the following structure:

In section II, it defines a generalized wake-function for two point charges, a source charge $S$, that generates the wakefields and a test charge $T$ that experiences the wakefields. The generalized wake-function is independent of the fact that the charges are countermoving or comoving and can describe what happens when the two beams are passing into the device at the same time. The section also introduces the generalized wake-potential and uses it to calculate an expression of the energy and the power dissipated by two countermoving beams transiting in a vacuum chamber, both in terms of wake-functions and in terms of impedances.

In Sec. III, the proposed model is benchmarked in the longitudinal plane, since this study focuses on beam energy loss and rf-heating. In particular, in Sec. III A, the longitudinal comoving and countermoving wake-function, wake-potential and impedance, computed analytically, are compared against the ones obtained by simulations. This is done for two geometries: a resistive wall pipe and a pillbox cavity. For both the examples, first the methodology and the results are shown (in Sec. III A 1 for the resistive wall pipe and in Sec. III A 2 for the pillbox cavity). Subsequently, the physical meaning of the results and their implication are discussed in the discussion Sec. III A 3 both for the resistive wall pipe and for the pillbox cavity. Section III A shows that there is good agreement between the proposed model results and the simulation ones.

In Sec. III B, the problem of the energy dissipated by two countermoving beams exciting and interacting with a pillbox cavity mode is tackled using both simulations and the proposed model. This subsection is divided in two parts. In the first part (Sec. III B 1), the methodology is described and the results are presented. In the second part (Sec. III B 2), the physical meaning of the results is discussed. Also Sec. III $B$ finds analytic and simulation results in good agreement. 
Finally, conclusions and future works are reported in Sec. IV.

\section{THE PHYSICAL MODEL}

\section{A. Main approximations}

The approximations on which the wakefield model is based are two-fold [3]:

(i) Rigid beam approximation. The trajectories of $S$ and $T$ are fixed, they are straight and parallel to each other. Furthermore, the speed modulus of $T$ and $S$ is equal and constant $v_{q_{S}}=\beta_{q_{S}} c=v_{q_{T}}=\beta_{q_{T}} c=v$ while the two particles traverse the vacuum chamber.

(ii) Kick approximation. The effects of the electromagnetic force, continuously acting on $S$ and $T$ all along the vacuum chamber, are represented as a lumped single change in particle properties acting after the particles passage.

Often in the literature the first hypothesis is reformulated as follows: the trajectories of $S$ and $T$ are fixed, they are straight and parallel to each other and the longitudinal relative position of $T$ with respect to $S$ (represented as $s_{S T}$ in Fig. 1(a)) during the particle transit through the vacuum chamber is constant, i.e., time-independent.

The authors want to stress that the previous sentence is not the rigid beam approximation but only one of its consequences. The time independence of the relative position of $T$ with respect to $S$ can be derived from the rigid beam approximation adding the extra hypothesis that $T$ and $S$ move in the same direction, that is the comoving case. The rigid beam approximation, as stated in this paper, remains valid also if the relative positions between the particles is changing inside the vacuum chamber. This is the case for the countermoving wakefield scenario, where the particle distance $s_{S T}$ changes while $T$ and $S$ are traversing the vacuum chamber, i.e., $s_{S T}$ is time dependent [refer to Fig. 1(b)].

In addition to the classical rigid beam and kick approximations, other two approximations are considered in this work.

It is assumed that all the interactions happen in the vacuum chamber. Electromagnetic fields generated by the beams outside of the vacuum chamber that reach the vacuum chamber and deposit energy on its wall are neglected.

Furthermore, when two countermoving beams are transiting in the same vacuum chamber at the same time, direct electromagnetic forces between the two beams develop. This interaction, known as beam-beam effects [17], is crucial to study beam dynamics and instabilities during collisions. However, it has negligible effects on the rf-heating of the vacuum chamber and it is not taken into account in this paper. As a consequence, the motion of the beams is considered not perturbed by the direct interactions between the beams and the rigid beam approximation can be applied.

The formal physical model that describes quantitatively the effects of the wakefield is well know and tested for the comoving case. However, this model relies on the time independence of the longitudinal test-source distance $s_{S T}$.

In the countermoving case, since $s_{S T}$ is not constant, the current model is not applicable as it is. However, it can be adapted to describe both the comoving and countermoving cases, as is explained in the following.

\section{B. Generalized wake-function and wake-potential}

In this subsection the expression of the generalized wake-function and wake-potential are introduced. Generalized means that they can be used both for the comoving and countermoving cases.

Primarily, one notes that there is a time delay for the entrance in the vacuum chamber of the test charge $T$ with respect to the source charge $S$. In this paper, this is called time entrance delay and it is defined as:

$$
\Delta t_{S T}=t_{T i}-t_{S i},
$$

where $t_{T i}$ is the entrance time of the test particle into the vacuum chamber and $t_{S i}$ is the entrance time of the source particle. For the sake of clarity, the test particle enters into the vacuum chamber when it crosses the test entrance section. The source particle enters into the vacuum chamber when it crosses the source entrance section, refer to Fig. 2. One also defines the space entrance delay as the distance that $T$ has to cover to enter into the vacuum chamber at the time at which $S$ is entering into the vacuum chamber:

$$
\Delta s_{S T}=v \Delta t_{S T} .
$$

In the comoving case the space entrance delay is coincident with the longitudinal distance between $T$ and $S$ and the concepts of time entrance delay, space entrance delay and longitudinal particle distance can be used indifferently. This is not the case in the countermoving scenario.

Subsequently, to formally define the wake-function, one has to recall its physical meaning: the wake-function represents the integrated effect (change of energy in the longitudinal direction and change of transverse momentum in the transverse plane) that the electromagnetic field excited by the transit of the source charge $S$ in the vacuum chamber has on the test charge $T$ that enters the vacuum chamber with a time delay $\Delta t_{S T}$ with respect to $S$.

Thus, a generalized wake-function can be defined as:

$\mathbf{w}\left(\mathbf{u}_{T}, \mathbf{u}_{S}, \Delta t_{S T}\right)=\frac{1}{q_{S} q_{T}} \int_{t_{T i}}^{t_{T o}} \mathbf{F}\left(\mathbf{u}_{T}, \mathbf{u}_{S}, \Delta t_{S T}, t\right) v d t$,

where, $\mathbf{F}$ is the instantaneous Lorentz force acting on $\mathrm{T}$ and $t_{T o}$ is the exit time of the test particle from the vacuum chamber. The vectors $\mathbf{u}_{T}, \mathbf{u}_{S}$ define the transverse positions of test and source particle trajectory (they are shown in Fig. 1).

The generalized wake-function $\mathbf{w}$ defined by Eq. (3) is a vector of three components. The longitudinal wake-function depends on the longitudinal electric field $E_{z}$ as: 


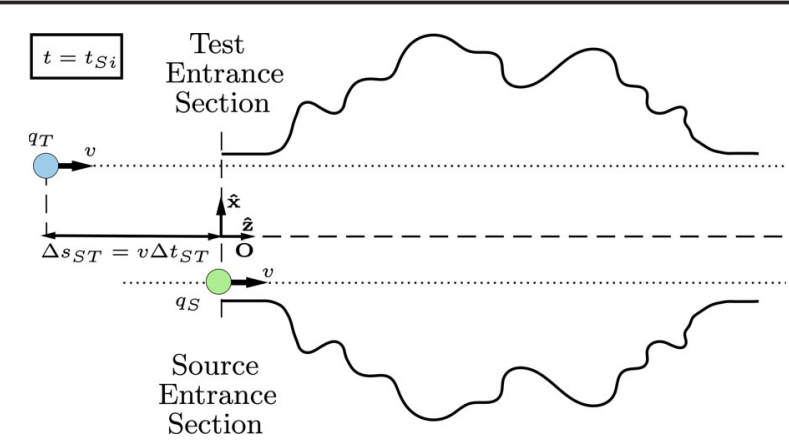

(a)

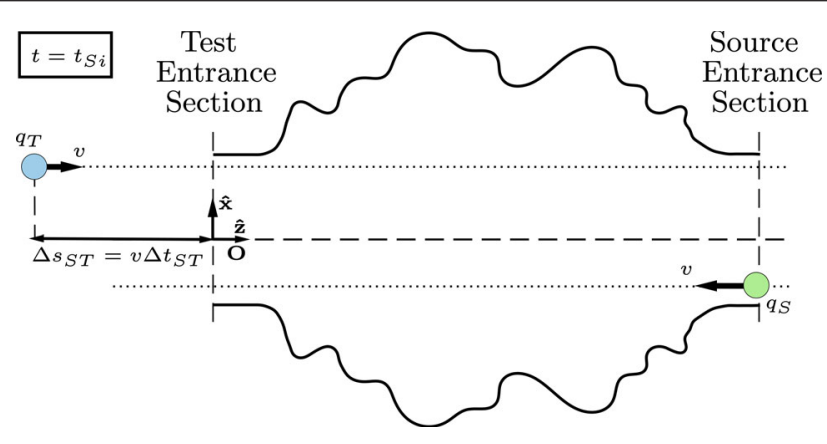

(b)

FIG. 2. Representation of the positions of $S$ and $T$ at the time at which the source is entering into the vacuum chamber, $t_{S i}$. Test and source entrance section are highlighted, in the comoving case they are coincident. The space entrance delay $\Delta s_{S T}$ is also represented, it is the distance that $T$ has to cover to enter into the vacuum chamber starting from the time when $S$ is entering. Note that, in the comoving case the space entrance delay is also the distance between the source and the test particle. (a) Comoving case. (b) Countermoving case.

$$
w_{z}\left(\mathbf{u}_{T}, \mathbf{u}_{S}, \Delta t_{S T}\right)=\frac{1}{q_{S}} \int_{t_{T i}}^{t_{T o}} E_{z}\left(\mathbf{u}_{T}, \mathbf{u}_{S}, \Delta t_{S T}, t\right) v d t
$$

Equation (4) will be used in this paper to give examples of countermoving wake-functions.

From Eq. (3) it is possible to obtain the generalized wake-potential. If one considers as a source a longitudinal charge distribution $\lambda_{S}(t)$, normalized such that $\int_{-\infty}^{\infty} \lambda_{S}(t) d t=1$, each infinitesimal elements of the charge distribution can be thought of as a point charge that enters into the vacuum chamber at a time $t_{d Q_{s} i}$ and has a different entrance delay with respect to the test charge $\mathrm{T}$, given by the equation $\Delta t_{S T}=t_{T i}-t_{d Q_{S} i}$. The charge of this infinitesimal element can be expressed as $d Q_{S}\left(t_{d Q_{S} i}\right)=$ $q_{S} \lambda_{S}\left(t_{d Q_{S} i}\right) d t_{d Q_{S} i}$. It is also useful to define $\Delta t_{Q_{S} T}$, the time entrance delay between the source distribution $Q_{S}$ and the test charge $T$ as the entrance delay between the test charge and a reference point charge in the distribution, $d Q_{S r}$ (for instance the infinitesimal element with the maximum charge). The space entrance delay between the source distribution $Q_{S}$ and the test charge T, $\Delta s_{Q_{S} T}$, follows from the time entrance delay as $\Delta s_{Q_{S} T}=v \Delta t_{Q_{S} T}$.

A visualization of these quantities is given in Fig. 3. The figure represents the sliced charge distribution $Q_{S}$ and the test charge $q_{T}$ as a function of their entrance times into the vacuum chamber $t_{i}$.

Every charge $d Q_{S}$ excites the vacuum chamber and acts on $T$ with its own wakefield. The sum of all these contribution is the wake-potential. Thus, the generalized wake-potential can be defined as the convolution between the generalized wake-function $\mathbf{w}$ and the normalized charged distribution $\lambda_{S}$ :

$\mathbf{W}\left(\mathbf{u}_{S}, \mathbf{u}_{T}, t_{T i}\right)=\int_{-\infty}^{\infty} \lambda_{S}\left(t_{d Q_{S} i}\right) \mathbf{w}\left(\mathbf{u}_{T}, \mathbf{u}_{S}, t_{T i}-t_{d Q_{S} i}\right) d t_{d Q_{S} i}$

In section III examples of longitudinal countermoving wake-functions and wake-potentials are reported.

\section{Energy and power}

The longitudinal component of the generalized wakefunction, $w_{z}$ expressed in Eq. (4), represents the energy variation of the test point charge because of the wakefield induced by the source point charge, normalized with respect to the two charges $q_{S}, q_{T}$. The longitudinal component of the generalized wake-potential, $W_{z}$ Eq. (5), represents the energy variation of the test point charge because of the wakefield induced by the source charge distribution, normalized with respect to the two total charges $q_{S}, q_{T}$.

If also the test charge is a distribution, it can be described as an ensemble of point charges, each one centered at its entrance time in the vacuum chamber $t_{d Q_{T} i}$ with width $d t_{d Q_{T} i}$ and charge $d Q_{T}\left(t_{d Q_{T} i}\right)=q_{T} \lambda_{T}\left(t_{d Q_{T} i}\right) d t_{d Q_{T} i}$.

The energy variation of the test distribution because of the wakefield induced by the source distribution, $\Delta U_{Q_{T} Q_{S}}$, is the sum of the energy variations of all the infinitesimal charges $d Q_{T}$, each one characterized by a different entrance time $t_{d Q_{T} i}$ :

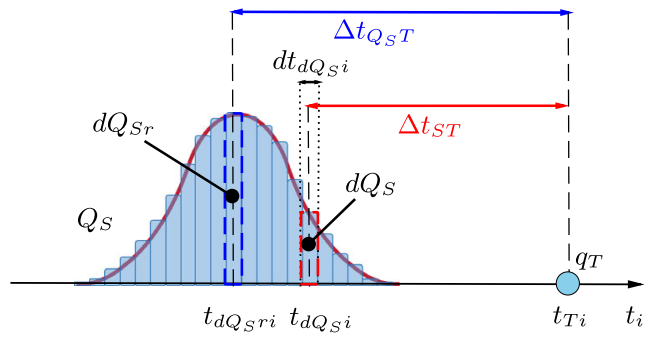

FIG. 3. Representation of the charge distribution $Q_{S}$ and of the test charge $q_{T}$ as a function of their entrance time into the vacuum chamber $t_{i}$. In the picture, $t_{d Q_{S i}}$ is the entrance time of $d Q_{S}$, the generic infinitesimal charge composing the distribution $Q_{S}, t_{d Q_{S r i}}$ is the entrance time of $d Q_{S r}$, the reference infinitesimal charge of the distribution $Q_{S}$. The entrance time delay of the test charge $q_{T}$ with respect to this two charges, $\Delta t_{S T}$ and $\Delta t_{Q_{S} T}$, is also shown. 


$$
\Delta U_{Q_{T} Q_{S}}=q_{S} q_{T} \int_{-\infty}^{\infty} \lambda_{T}\left(t_{d Q_{T} i}\right) W_{z}\left(\mathbf{u}_{T}, \mathbf{u}_{S}, t_{d Q_{T} i}\right) d t_{d Q_{T} i} .
$$

Considering a characteristic frequency $f_{0}$, as, for example, the beam revolution frequency in the accelerator (and a related characteristic time $T_{0}=1 / f_{0}$ ), one can define the average power gain or loss of the test distribution transiting into the vacuum chamber as:

$$
P=\Delta U_{Q_{T} Q_{S}} f_{0}=\frac{\Delta U_{Q_{T} Q_{S}}}{T_{0}} .
$$

\section{Energy dissipation due to two beams traversing a vacuum chamber}

In this section the longitudinal generalized wakefunction and wake-potential presented earlier are used to obtain the expressions of the beam energy and power variation, in the case of two beams traversing the same vacuum chamber. These expressions are valid both for comoving and countermoving beams.

When two beams traverse the same vacuum chamber, they act as sources and tests at the same time: they both generate wakefields (source behavior) that act back on themselves and on the other beam (test behavior).

To model this situation, with reference to Fig. 4, beam one and beam two are introduced. They have a total charge $q_{1}$ and $q_{2}$ and longitudinal distributions $\lambda_{\circ 1}(t)$ and $\lambda_{\circ 2}(t)$.

If one distribution is thought of as source, the linked quantities have the subscript $S$ instead of "॰," as example $Q_{S 1}(t)$ instead of $Q_{\circ 1}(t)$. If one distribution is thought of as test, the linked quantities have the subscript $T$.

The rigid beam approximation is still valid, i.e., the two beams are moving on straight and parallel trajectories and the speed of the particles is preserved during the traversal.

The time at which beam one and beam two enter in the vacuum chamber, $t_{1 i}$ and $t_{2 i}$ respectively, is defined as the time at which the center of the distribution crosses the relative beam entrance section. The beam entrance sections for two countermoving beams are reported in Fig. 4. In the case of comoving beams, the beam entrance sections would be coincident and located at one side of the vacuum chamber.

Thus, the time entrance delay between the beams can be defined as:

$$
\Delta t_{b_{1} b_{2}}=t_{2 i}-t_{1 i}
$$

for a positive value of $\Delta t_{b_{1} b_{2}}$ beam two is delayed with respect to beam one, and vice versa for a negative value.

\section{Energy variation in time domain}

The total energy variation of the two beam system, $\Delta U$, is the sum of the energy lost or gained by beam one, $\Delta U_{1}$, plus the energy lost or gained by beam two, $\Delta U_{2}$ :

$$
\Delta U=\Delta U_{1}+\Delta U_{2} .
$$

The energy variation of beam one is due to the effects of its own wakefield plus the effect of the wakefield generated by beam two. Beam two also experiences the wakefield generated by beam one plus its own wakefield, thus, one can further split the terms in Eq. (9) as:

$$
\begin{aligned}
& \Delta U_{1}=\Delta U_{Q_{S 1} Q_{T 1}}+\Delta U_{Q_{S 2} Q_{T 1}}, \\
& \Delta U_{2}=\Delta U_{Q_{S 1} Q_{T 2}}+\Delta U_{Q_{S 2} Q_{T 2}} .
\end{aligned}
$$

In Eqs. (10) and (11), $\Delta U_{Q_{S n} Q_{T m}}$, is the energy variation of beam $m$ because of the wakefield generated by beam $n$. The case of two countermoving beams, with $n=m$, can be treated as a comoving wakefield scenario. If $n \neq m$, it can be treated as a countermoving wakefield scenario. The case of two comoving beams, independently of $n$ and $m$, can be treated as a comoving wakefield scenario.

A case of interest is the one where the two beams have the same structure, i.e., they are described by the same distribution $\lambda$ (this is the scenario in the LHC for protonproton and ion-ion collision [6]). With this hypothesis, let us consider a time reference frame in which the origin is

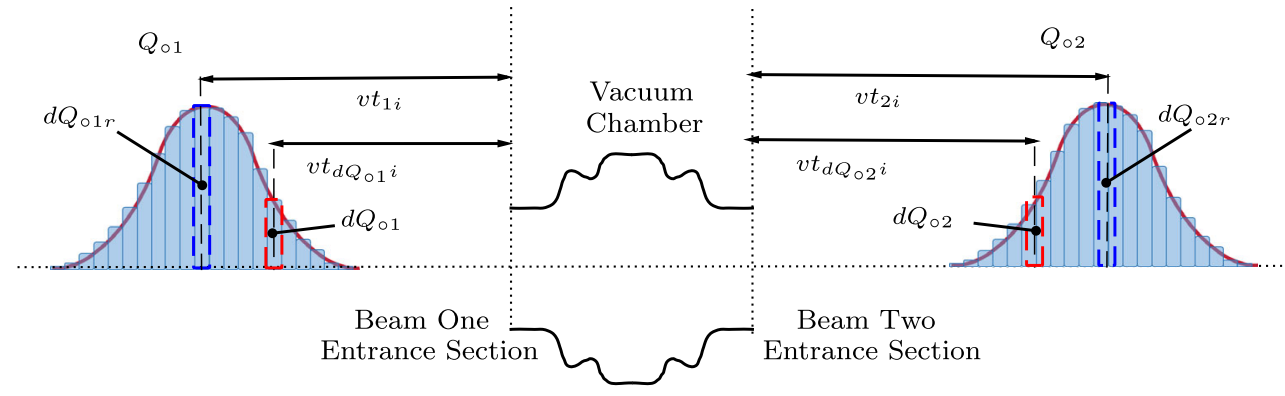

FIG. 4. A vacuum chamber and two countermoving beam distributions, $Q_{\circ 1}$ and $Q_{\circ 2}$, are represented in space as two Gaussian bunches. Both the distributions, divided in infinitesimal charges $d Q_{\circ 1}$ and $d Q_{\circ 2}$, are moving to enter inside the vacuum chamber. The beam entrance times in the vacuum chamber $t_{1 i}$ and $t_{2 i}$ as well as the generic entrance time of the generic infinitesimal charge, $t_{d Q_{\circ 1} i}$ and $t_{d Q_{\circ 2} i}$ are also shown. 
set such that $t_{1 i}=0$, i.e., the time origin is set at the moment in which beam one is entering into the vacuum chamber. Since there is a delay $\Delta t_{b_{1} b_{2}}$ between the two beams, in this time reference frame one has:

$$
\begin{aligned}
& \lambda_{T 1}\left(t_{d Q_{T 1} i}\right)=\lambda\left(t_{d Q_{T 1} i}\right), \\
& \lambda_{S 1}\left(t_{d Q_{S 1} i}\right)=\lambda\left(t_{d Q_{S 1} i}\right), \\
& \lambda_{S 2}\left(t_{d Q_{S 2} i}\right)=\lambda\left(t_{d Q_{S 2} i}-\Delta t_{b_{1} b_{2}}\right) .
\end{aligned}
$$

Similar equations can be obtained considering a time reference frame which origin is set such that $t_{S 2 i}=0$, i.e., the time origin is set at the moment in which beam two is entering into the vacuum chamber.
They are:

$$
\begin{aligned}
& \lambda_{T 2}\left(t_{d Q_{T 2} i}\right)=\lambda\left(t_{d Q_{T 2} i}\right), \\
& \lambda_{S 2}\left(t_{d Q_{S 2} i}\right)=\lambda\left(t_{d Q_{S 2} i}\right), \\
& \lambda_{S 1}\left(t_{d Q_{S 1} i}\right)=\lambda\left(t_{d Q_{S 1} i}+\Delta t_{b_{1} b_{2}}\right) .
\end{aligned}
$$

It is more convenient to compute the energy variation of beam one, $\Delta U_{1}$, in the reference frame with $t_{1 i}=0$ and the energy variation of beam two, $\Delta U_{2}$, in the reference frame with $t_{2 i}=0$. Thus, substituting Eqs. (12) and (13) into the energy variation expressions, Eq. (6), where also Eq. (5) has been considered, one obtains:

$$
\begin{aligned}
& \Delta U_{Q_{S 1} Q_{T 1}}=q_{1}^{2} \int_{-\infty}^{\infty} \lambda\left(t_{d Q_{T 1} i}\right) \int_{-\infty}^{\infty} \lambda\left(t_{d Q_{S 1} i}\right) w_{z 11}\left(t_{d Q_{T 1} i}-t_{d Q_{S 1} i}\right) d t_{d Q_{S 1} i} d t_{d Q_{T 1} i}, \\
& \Delta U_{Q_{S 2} Q_{T 1}}=q_{2} q_{1} \int_{-\infty}^{\infty} \lambda\left(t_{d Q_{T 1} i}\right) \int_{-\infty}^{\infty} \lambda\left(t_{d Q_{S 2} i}-\Delta t_{b_{1} b_{2}}\right) w_{z 21}\left(t_{d Q_{T 1} i}-t_{d Q_{S 2} i}\right) d t_{d Q_{S 2} i} d t_{d Q_{T 1} i}, \\
& \Delta U_{1}=q_{1}^{2} \int_{-\infty}^{\infty} \lambda\left(t_{d Q_{T 1} i}\right)\left[\int_{-\infty}^{\infty} \lambda\left(t_{d Q_{S 1} i}\right) w_{z 11}\left(t_{d Q_{T 1} i}-t_{d Q_{S 1} i}\right) d t_{d Q_{S 1} i}\right. \\
& \left.+\frac{q_{2}}{q_{1}} \int_{-\infty}^{\infty} \lambda\left(t_{d Q_{S 2} i}-\Delta t_{b_{1} b_{2}}\right) w_{z 21}\left(t_{d Q_{T 1} i}-t_{d Q_{S 2} i}\right) d t_{d Q_{S 2} i}\right] d t_{d Q_{T 1} i} \\
& \Delta U_{Q_{S 2} Q_{T 2}}=q_{2}^{2} \int_{-\infty}^{\infty} \lambda\left(t_{d Q_{T 2} i}\right) \int_{-\infty}^{\infty} \lambda\left(t_{d Q_{S 2} i}\right) w_{z 22}\left(t_{d Q_{T 2} i}-t_{d Q_{S 2} i}\right) d t_{d Q_{S 2} i} d t_{d Q_{T 2} i}, \\
& \Delta U_{Q_{S 1} Q_{T 2}}=q_{1} q_{2} \int_{-\infty}^{\infty} \lambda\left(t_{d Q_{T 2} i}\right) \int_{-\infty}^{\infty} \lambda\left(t_{d Q_{S 1} i}+\Delta t_{b_{1} b_{2}}\right) w_{z 12}\left(t_{d Q_{T 2} i}-t_{d Q_{S 1} i}\right) d t_{d Q_{S 1} i} d t_{d Q_{T 2} i}, \\
& \Delta U_{2}=q_{2}^{2} \int_{-\infty}^{\infty} \lambda\left(t_{d Q_{T 2} i}\right)\left[\int_{-\infty}^{\infty} \lambda\left(t_{d Q_{S 2} i}\right) w_{z 22}\left(t_{d Q_{T 2} i}-t_{d Q_{S 2} i}\right) d t_{d Q_{S 2} i}\right. \\
& \left.+\frac{q_{1}}{q_{2}} \int_{-\infty}^{\infty} \lambda\left(t_{d Q_{S 1} i}+\Delta t_{b_{1} b_{2}}\right) w_{z 12}\left(t_{d Q_{T 2} i}-t_{d Q_{S 1} i}\right) d t_{d Q_{S 1} i}\right] d t_{d Q_{T 2} i}
\end{aligned}
$$

In Eqs. (14)-(19), the terms $w_{z n m}$ represent the longitudinal wake-function of the beam $n$ seen as source on the beam $m$ seen as test.

Considering Eq. (9), to get the total energy loss by the two beams, $\Delta U$, one has to sum the contributions of beam one and beam two, $\Delta U_{1}$ and $\Delta U_{2}$. It is important to stress that this energy variation is independent of the origin of the time reference frame. Indeed, it represents the work done by the electric field in the vacuum chamber (the wakefield) on the beams. Thus, it is irrelevant if $\Delta U_{1}$ has been computed in a different time frame than $\Delta U_{2}$.

A quantity that is used in the following, directly linked to the energy variation, is the beam loss factor $k$. It quantifies the energy variation of a beam normalized by the square of the beam charge. In the case of two beams one has:

$$
k_{1}=\frac{\Delta U_{1}}{q_{1}^{2}}, \quad k_{2}=\frac{\Delta U_{2}}{q_{2}^{2}},
$$

for beam one and beam two respectively.

\section{Energy variation in the frequency domain}

If a Fourier transform is applied to Eq. (16) and Eq. (19) one obtains the expressions of the energy variation of each beam in the frequency domain. From Eq. (9), the expression of the total energy variation of the two beam system in the frequency domain is computed. Thus, 
one has:

$$
\begin{aligned}
& \Delta U=\frac{1}{\pi} \int_{0}^{\infty}|\Lambda(\omega)|^{2}\left\{q_{1}^{2} \operatorname{Re}\left[Z_{11}\right]+q_{2}^{2} \operatorname{Re}\left[Z_{22}\right]+q_{1} q_{2} \operatorname{Re}\left[Z_{12}\right] \cos \left(\omega \Delta t_{b_{1} b_{2}}\right)\right. \\
& \left.+q_{1} q_{2} \operatorname{Re}\left[Z_{21}\right] \cos \left(\omega \Delta t_{b_{1} b_{2}}\right)+q_{1} q_{2} \operatorname{Im}\left[Z_{12}\right] \sin \left(\omega \Delta t_{b_{1} b_{2}}\right)-q_{1} q_{2} \operatorname{Im}\left[Z_{21}\right] \sin \left(\omega \Delta t_{b_{1} b_{2}}\right)\right\} d \omega, \\
& \Delta U_{1}=\frac{q_{1}^{2}}{\pi} \int_{0}^{\infty}|\Lambda(\omega)|^{2}\left\{\operatorname{Re}\left[Z_{11}\right]+\frac{q_{2}}{q_{1}} \operatorname{Re}\left[Z_{21}\right] \cos \left(\omega \Delta t_{b_{1} b_{2}}\right)-\frac{q_{2}}{q_{1}} \operatorname{Im}\left[Z_{21}\right] \sin \left(\omega \Delta t_{b_{1} b_{2}}\right)\right\} d \omega, \\
& \Delta U_{2}=\frac{q_{2}^{2}}{\pi} \int_{0}^{\infty}|\Lambda(\omega)|^{2}\left\{\operatorname{Re}\left[Z_{22}\right]+\frac{q_{1}}{q_{2}} \operatorname{Re}\left[Z_{12}\right] \cos \left(\omega \Delta t_{b_{1} b_{2}}\right)+\frac{q_{1}}{q_{2}} \operatorname{Im}\left[Z_{12}\right] \sin \left(\omega \Delta t_{b_{1} b_{2}}\right)\right\} d \omega,
\end{aligned}
$$

where $\Lambda$ is the Fourier transform of the time charge distribution $\lambda, Z_{n m}$ is the longitudinal impedance, Fourier transform of the longitudinal wake-functions $w_{z n m}, \operatorname{Re}[\cdot]$ and $\operatorname{Im}[\cdot]$ are the real and imaginary part operators, $\omega=2 \pi f$ with $f$ the generic frequency.

A case of interest is when the two beams enter and traverse the vacuum chamber repeatedly with a characteristic frequency $f_{0}$ (in a collider this is the beam revolution frequency).

In this case, applying the procedure described in the work of Rumolo [18] to Eqs. (21)-(23), one can write:

$$
\begin{gathered}
\Delta U=\frac{f_{0}}{\pi} \sum_{p=-\infty}^{\infty}\left|\Lambda\left(p \omega_{0}\right)\right|^{2}\left\{q_{1}^{2} \operatorname{Re}\left[Z_{11}\right]+q_{2}^{2} \operatorname{Re}\left[Z_{22}\right]+q_{1} q_{2} \operatorname{Re}\left[Z_{12}\left(p \omega_{0}\right)\right] \cos \left(p \omega_{0} \Delta t_{b_{1} b_{2}}\right)\right. \\
+q_{1} q_{2} \operatorname{Re}\left[Z_{21}\left(p \omega_{0}\right)\right] \cos \left(p \omega_{0} \Delta t_{b_{1} b_{2}}\right)+q_{1} q_{2} \operatorname{Im}\left[Z_{12}\left(p \omega_{0}\right)\right] \sin \left(p \omega_{0} \Delta t_{b_{1} b_{2}}\right) \\
\left.-q_{1} q_{2} \operatorname{Im}\left[Z_{21}\left(p \omega_{0}\right)\right] \sin \left(p \omega_{0} \Delta t_{b_{1} b_{2}}\right)\right\} \\
\Delta U_{1}=\frac{f_{0} q_{1}^{2}}{\pi} \sum_{p=-\infty}^{\infty}\left|\Lambda\left(p \omega_{0}\right)\right|^{2}\left\{\operatorname{Re}\left[Z_{11}\left(p \omega_{0}\right)\right]+\frac{q_{2}}{q_{1}} \operatorname{Re}\left[Z_{21}\left(p \omega_{0}\right)\right] \cos \left(p \omega_{0} \Delta t_{b_{1} b_{2}}\right)-\frac{q_{2}}{q_{1}} \operatorname{Im}\left[Z_{21}\left(p \omega_{0}\right)\right] \sin \left(p \omega_{0} \Delta t_{b_{1} b_{2}}\right)\right\} \\
\Delta U_{2}=\frac{f_{0} q_{2}^{2}}{\pi} \sum_{p=-\infty}^{\infty}\left|\Lambda\left(p \omega_{0}\right)\right|^{2}\left\{\operatorname{Re}\left[Z_{22}\right]+\frac{q_{1}}{q_{2}} \operatorname{Re}\left[Z_{12}\left(p \omega_{0}\right)\right] \cos \left(p \omega_{0} \Delta t_{b_{1} b_{2}}\right)+\frac{q_{1}}{q_{2}} \operatorname{Im}\left[Z_{12}\left(p \omega_{0}\right)\right] \sin \left(p \omega_{0} \Delta t_{b_{1} b_{2}}\right)\right\}
\end{gathered}
$$

where $\omega_{0}=2 \pi f_{0}$. Finally, using Eq. (7), one can transform Eqs. (14)-(26) from energy variation to power variation.

It is important to notice that Eqs. (14)-(19) and (21)-(26) are valid both for countermoving and comoving beams. Indeed, no restrictive assumption on the propagation direction of the single beam is made in the derivation of the equations, since the information on the beam direction is provided by the wake-functions and the corresponding impedances.

In this paper, $Z_{11}$ and $Z_{22}$ are labeled comoving impedances and $Z_{12}$ and $Z_{21}$ countermoving ones.

Equations (21)-(26) show that the total energy lost by two beams in a vacuum chamber, $\Delta U$, and the energy variation of each beam, $\Delta U_{1}$ and $\Delta U_{2}$, depend on the real part of the beam comoving impedances, $Z_{11}$ and $Z_{22}$, as expected, and on both the real and imaginary parts of the countermoving impedances, $Z_{12}$ and $Z_{21}$. Furthermore, the dependency of the energy variations with the entrance delay between the beams, $\Delta t_{b_{1} b_{2}}$, is isolated in a sine and a cosine function multiplying the real and imaginary parts of the countermoving impedances. This equation structure leads to an interference like behavior of the total energy as a function of the entrance delay between the beams. This is shown with an example in Sec. III B, where Eqs. (21)-(23) are applied to compute the energy variation of the single beams and the total energy variation of the two beams. In the discussion part of that section a possible explanation of the interference behavior is provided.

Finally, the authors want to stress that Eqs. (21) and (24) express the energy loss of the two beam system transiting a vacuum chamber due to the wakefield generation. Generally speaking, wakefields dissipate only a part of their energy in the vacuum chamber as heat. Some 
wakefields can escape the vacuum chamber in the form of untrapped electromagnetic modes.

However, in hadron machines, since the cutoff frequency of the pipe is generally above the beam frequency content, the wakefields generated by the beams during their passage in the vacuum chamber remain trapped therein. The wakefield energy is entirely dissipated on the vacuum chamber as heat. Thus, for the majority of the hadron machines, one can also say that Eqs. (21) and (24) provide the energy dissipated as heat on the vacuum chamber due to the passage of two beams. Analogously, Eq. (7) express the heating source power of the vacuum chamber due to the passage of two beams.

\section{E. Effects of the entrance delay on the total energy losses}

It is evident from Eq. (21) or (24) that the total energy lost by the two beams in the vacuum chamber is dependent on the entrance delay between the beams, i.e., $\Delta t_{b_{1} b_{2}}$. It is interesting to understand the values of $\Delta t_{b_{1} b_{2}}$ for which the energy lost by the two beams has a maximum or a minimum. This is done by differentiating Eq. (21) or (24) with respect to $\Delta t_{b_{1} b_{2}}$, imposing the result equal to zero and solving the resultant equation. For Eq. (21) this leads to:

$\Delta t_{b_{1} b_{2}}=\frac{1}{\omega} \operatorname{atan}\left(\frac{\operatorname{Im}\left[Z_{21}(\omega)\right]-\operatorname{Im}\left[Z_{12}(\omega)\right]}{\operatorname{Re}\left[Z_{21}(\omega)\right]+\operatorname{Re}\left[Z_{12}\right](\omega)}\right)+\frac{n \pi}{\omega}$

where $n \in \mathbb{N}$ and $n \geq 0$.

For vacuum chambers that are longitudinally symmetric (i.e., they have a symmetry plane perpendicular to the propagation direction of the beams), if the beams trajectories are superimposed, the relation $Z_{12}=Z_{21}$ has to hold because the two beams are completely interchangeable. In this case Eq. (27) is reduced to:

$$
\Delta t_{b_{1} b_{2}}=\frac{n \pi}{\omega}=\frac{n}{2 f}
$$

where $\omega=2 \pi f$ has been used, with $f$ the generic frequency.

In Sec. III B, Eq. (28) is used to compute the entrance delay between two countermoving beams that leads to the maximum and the minimum value of the energy dissipated in a pillbox cavity.

\section{RESULTS}

\section{A. Model benchmark for the calculation of longitudinal countermoving wake-functions and wake-potentials}

In this subsection the formalism developed in the previous section is benchmarked. The comoving and countermoving longitudinal wake-potentials obtained using the previously outlined model are compared to the ones obtained by simulations. This is done for two example geometries: a resistive wall pipe and a pillbox cavity.
The general formalism developed in the previous section is applied, first to obtain again the well-known results for the comoving wake-potential, then to obtain the countermoving wake-potential. The wake-potentials are computed starting from the expression of the longitudinal electric field in the studied structures. Then, they are compared against the results of the CST Studio Suite 2018 commercial software [19]. In the countermoving wakefield case, with this benchmark the authors want to validate the capability of the proposed model and of the CST software to describe the effects of two countermoving beams in one vacuum chamber. A further test on the CST software is illustrated in the Appendix where the Panofsky-Wenzel theorem [20] was verified for two countermoving beams.

When possible, the results of the proposed formalism and the numerical ones are compared also with other analytic expressions of the wake-potential available in the literature. All the examples shown in this paper consider relativistic particles, i.e., $v=c$ with $c$ the speed of light.

For the wake-potential computation, a beam distribution $\lambda_{S}$ composed by one bunch with a Gaussian shape was considered. This is the default excitation signal for CST and it made benchmarking analytic results against numerical ones easier.

Using Eq. (1) and setting the origin of the time reference frame such that $t_{S i}=0$, one has:

$$
\Delta t_{S T}=t_{T i},
$$

and consequently,

$$
t_{T o}=t_{T i}+\frac{L}{v}=\Delta t_{S T}+\frac{L}{v} .
$$

Finally, with reference to Fig. 1, for the comoving case one has:

$$
z_{q_{S}}(t)=v t, \quad z_{q_{T}}(t)=v t-v \Delta t_{S T},
$$

and, for the countermoving case:

$$
z_{q_{S}}(t)=L-v t, \quad z_{q_{T}}(t)=v t-v \Delta t_{S T} .
$$

\section{The resistive wall pipe, methodology and results}

The case of the resistive cylindrical pipe is shown in Fig. 5. The problem is extensively discussed by Chao [2], with a longitudinal electric field induced by an ultrarelativistic disk charge traversing an infinitely long pipe given by (for the classic thick wall regime):

$$
\begin{aligned}
E_{z}\left(z_{q_{T}}(t), z_{q_{S}}(t)\right)= & -\frac{4 q_{S}}{\pi \epsilon_{0} b^{2}}\left[\frac{1}{3} e^{u} \cos (\sqrt{3} u)\right. \\
& \left.-\frac{\sqrt{2}}{\pi} \int_{0}^{\infty} d x \frac{x^{2} e^{u x^{2}}}{x^{6}+8}\right],
\end{aligned}
$$

where, $b$ is the radius of the pipe and 


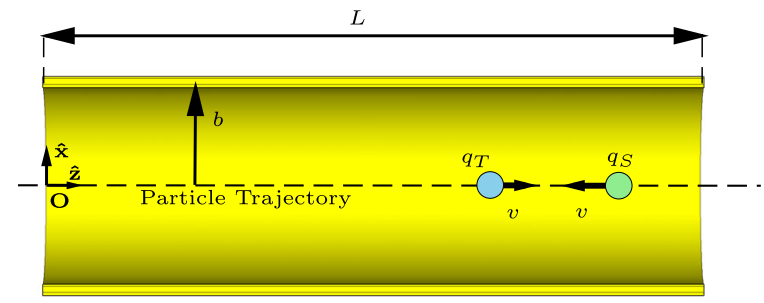

FIG. 5. Test and source charges traversing the resistive wall pipe at constant speed $v$. Countermoving case. The trajectory of both charges is coincident with the pipe axis.

$$
\begin{aligned}
u=-\psi \frac{s_{S T}(t)}{b(2 \chi)^{\frac{1}{3}}} & =-\psi \frac{z_{q_{S}}(t)-z_{q_{T}}(t)}{b(2 \chi)^{\frac{1}{3}}} \\
\chi & =\frac{c \varepsilon_{0}}{\sigma b}
\end{aligned}
$$

are two adimensional coefficients, $s_{S T}$ is the distance between $S$ and $T, \sigma$ is the conductivity of the pipe material and $\varepsilon_{0}$ is the dielectric vacuum constant. The term $\psi$ is not present in the work of Chao [2], it has been introduced to extend the validity of Eq. (33) also to the countermoving case. Its value is 1 for the comoving case scenario, while it is -1 for the countermoving case scenario. Furthermore, $u$ is defined with a minus sign with respect to Chao work [2] because of the different definition of distance between $S$ and $T$. Equation (33) holds for an infinitely long pipe, however, one can consider a pipe with finite length as a segment of this infinitely long pipe and compute the wakefunction on that part of the pipe. Using this approach, one has to be particularly careful on what happens at the boundaries, i.e., at the entrance and at the exit of the pipe. This point, and its implication on the results, will be discussed in Sec. III A 3.

For the comoving scenario, one substitutes Eq. (31) into Eq. (34), obtaining:

$$
u=-\frac{v \Delta t_{S T}}{b(2 \chi)^{\frac{1}{3}}} .
$$

Using Eq. (36) and Eq. (33), one obtains $E_{z}$, the longitudinal electric field experienced by the test charge as a function of the time entrance delay between source and test charge in the comoving case. Knowing $E_{z}$, the longitudinal wake-function is found by its definition, Eq. (4). Thus, also the wake-potential can be computed using Eq. (5).

For the countermoving scenario, one substitutes Eq. (32) into Eq. (34), obtaining:

$$
u=\frac{v\left(\Delta t_{S T}-2 t\right)+L}{b(2 \chi)^{\frac{1}{3}}} .
$$

As for the comoving case, Eq. (37) is substituted into Eq. (33) to obtain the longitudinal electric field $E_{z}$. Subsequently, using Eq. (4), the longitudinal wake-function can be obtained.
When using Eq. (4), since the particles are relativistic, attention has to be paid to the fact that there is no electromagnetic field in front of the particles and they do not interact before the meeting time $t_{m}$. The meeting time is defined as the time at which the longitudinal distance between the particles is null, i.e., $z_{q_{S}}\left(t_{m}\right)-z_{q_{T}}\left(t_{m}\right)=0$, so that $t_{m}=L / 2 v+\Delta t_{S T} / 2$. Thus, in the case of two countermoving relativistic particles Eq. (4) needs to be modified into:

$$
w_{z}=\left\{\begin{array}{ll}
\frac{v}{q_{S}} \int_{t_{T i}}^{t_{T o}} E_{z}\left(\Delta t_{S T}, t\right) d t & \text { if } t_{T i} \geq t_{m} \\
\frac{v}{q_{S}} \int_{t_{m}}^{t_{T o}} E_{z}\left(\Delta t_{S T}, t\right) d t & \text { if } t_{T i}<t_{m}
\end{array} .\right.
$$

Once the longitudinal wake-function is known the wakepotential can also be computed using Eq. (5).

Both in the comoving and in the countermoving case, Eqs. (38) and (5) were integrated numerically. In particular, the longitudinal wake-potential $w_{z}$ as a function of the time entrance delay $\Delta t_{S T}$ was obtained integrating Eq. (38) in $t$ for different values of $\Delta t_{S T}$. This could be done since $t_{T o}$ and $t_{T i}$ are expressed as a function of $\Delta t_{S T}$ by Eqs. (29) and (30).

A comparison between the comoving and countermoving wake-function is shown in Fig. 6 for a pipe with the following parameters: pipe length $L=0.3 \mathrm{~m}$, radius $b=0.05 \mathrm{~m}$, electric conductivity $\sigma=3.5 \times 10^{3} \mathrm{~S} \mathrm{~m}^{-1}$. A low value of $\sigma$ was imposed in order to obtain a large wakefield, so that the wake-potential estimation by simulations, presented later in this section, was less sensitive to numerical noise. The shape of the countermoving wakefunction is discussed in greater depth in Sec. III A 3.

In Fig. 7(a) the resistive pipe comoving wake-potentials, obtained by the previous formalism (indicated as semianalytic), by analytic formula [21,22] (indicated as Bane-Piwinski), and by CST Wakefield simulations (indicated as CST) are compared.

In Fig. 7(b) the resistive pipe countermoving wakepotentials obtained by the formalism developed in this

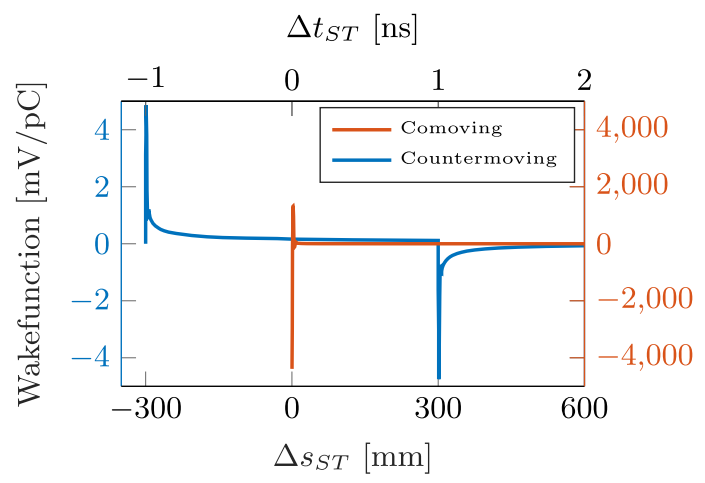

FIG. 6. Semi-analytic wake-functions of a point charge traversing a beam pipe. Pipe parameters: $b=0.05 \mathrm{~m}, \sigma=3.5 \times 10^{3} \mathrm{Sm}^{-1}$, $L=0.3 \mathrm{~m}$. For both particles $v=c$. Colors refer to different scales. 


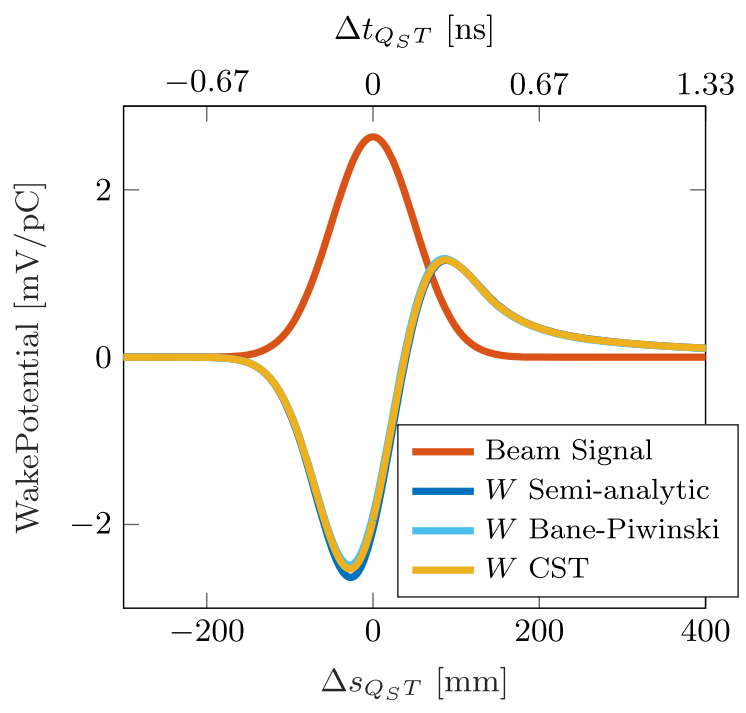

(a)

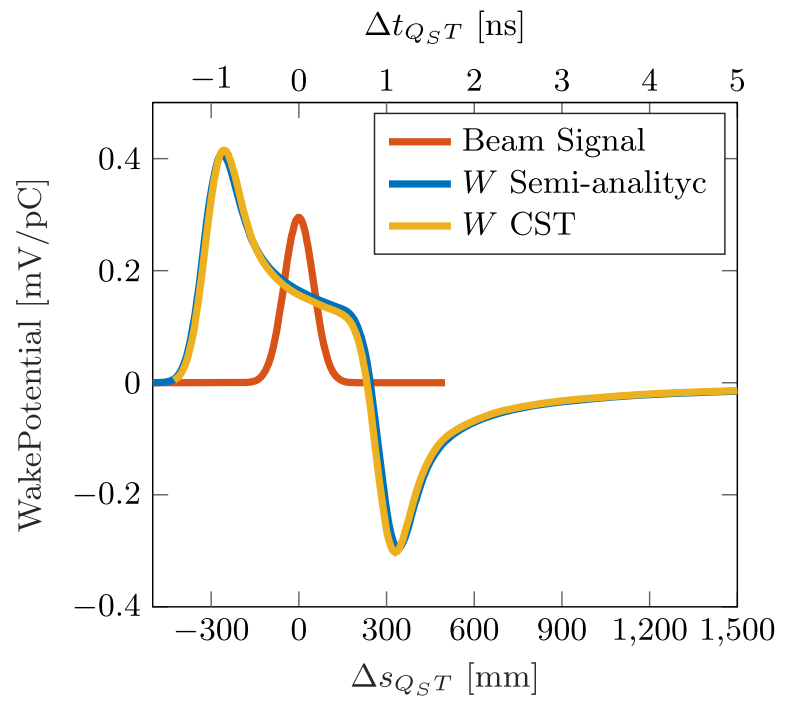

(b)

FIG. 7. Wakepotential of a Gaussian bunch profile traversing a beam pipe, comparison between semianalytic method and CST Wakefield solver. Pipe parameters: $b=0.05 \mathrm{~m}, \sigma=3.5 \times 10^{3} \mathrm{~S} \mathrm{~m}^{-1}, L=0.3 \mathrm{~m}$. Bunch parameters: $\sigma_{b}=0.05 \mathrm{~m}, v=c$. The beam signal is in arbitrary units for representational purposes. (a) Comoving wakepotential. (b) Countermoving wakepotential.

paper (indicated as semi-analytic), and by CST Wakefield simulations (indicated as CST) are compared.

In both the comoving and countermoving case there is an excellent agreement between the formalism and the simulation results.

\section{The pillbox cavity, methodology, and results}

This subsection obtains the longitudinal comoving and countermoving wake-function, impedance and wakepotential of a perfect electric conductor (PEC) pillbox cavity without incoming pipes. Contrary to the case of the pillbox with incoming pipes, an analytic expression of the electric field generated by a charge traveling inside a cavity without incoming pipes is known. Since this expression is crucial to obtain analytic results and a proper benchmark of simulation results the geometry without the incoming pipes was used.
In the pillbox cavity without incoming pipes only trapped modes can exist. This makes the example more representative for machines that have most of the beam power spectrum below the cutoff frequency of the beam pipe, as the majority of the hadron machines (like the LHC, which is the case of interest of this work). In these machines, a beam crossing a vacuum chamber excites only modes with frequencies in its power spectrum, that is below the cutoff frequency of the machine pipes. This means that, virtually all the excited modes remain trapped in the vacuum chamber, as it is the case for the proposed example.

The analytic expression of the transient longitudinal electric field generated by a disc of electrons moving from one side of a lossless pillbox cavity (radius $a$ and length $L$ ) to the other side at a speed $v_{S}=\beta_{S} c$, as shown in Figs. 8(a) and 8(b), was found by Faust [23] as:

$$
\begin{aligned}
E_{z}^{\prime}\left(r_{q_{T}}{ }^{\prime}, z_{q_{T}}{ }^{\prime}, t\right)= & -\frac{e N}{\epsilon_{0}}\left\{\frac{\beta_{S}}{L}\left[c t U(c t)-\left(c t-\frac{L}{\beta_{S}}\right) U\left(c t-\frac{L}{\beta_{S}}\right)\right]-U\left(c t-\frac{z_{q_{T}}^{\prime}}{\beta_{S}}\right)\right. \\
& -\frac{2 a \beta_{S}}{L} \sum_{m=1}^{m=\infty} \frac{J_{0}\left(r_{q_{T}}^{\prime} \rho_{m} / a\right)}{J_{1}\left(\rho_{m}\right) \rho_{m}^{2}}\left[\sin \left(\rho_{m} \frac{c t}{a}\right)-\sin \left(\rho_{m} \frac{c t-L / \beta_{S}}{a}\right) U\left(c t-\frac{L}{\beta_{S}}\right)\right] \\
& -\frac{4 a \beta_{S}}{L} \sum_{m=1}^{m=\infty} \sum_{n=1}^{n=\infty} \frac{J_{0}\left(r_{q_{T}}^{\prime} \rho_{m} / a\right)}{J_{1}\left(\rho_{m}\right)} \rho_{m} \frac{\sin \left(\gamma_{1} c t / a\right)-(-1)^{n} \sin \left(\gamma_{1} \frac{c t-L}{\beta_{S} a}\right) U\left(c t-\frac{L}{\beta_{S}}\right)}{\gamma_{1} \gamma_{2}^{2}} \cos \left(\frac{n \pi z_{q_{T}}^{\prime}}{L}\right) \\
& \left.+\frac{2}{\pi} \sum_{n=1}^{n=\infty} \frac{I_{0}\left(r_{q_{T}}^{\prime} n \pi \gamma_{S} / L\right)}{n I_{0}\left(a n \pi \gamma_{S} / L\right)}\left[\sin \left(n \pi \beta_{S} \frac{c t}{L}\right)-(-1)^{n} \sin \left(n \pi \beta_{S} \frac{c t-L / \beta_{S}}{L}\right) U\left(c t-\frac{L}{\beta_{S}}\right)\right] \cos \left(\frac{n \pi z_{q_{T}}^{\prime}}{L}\right)\right\},
\end{aligned}
$$




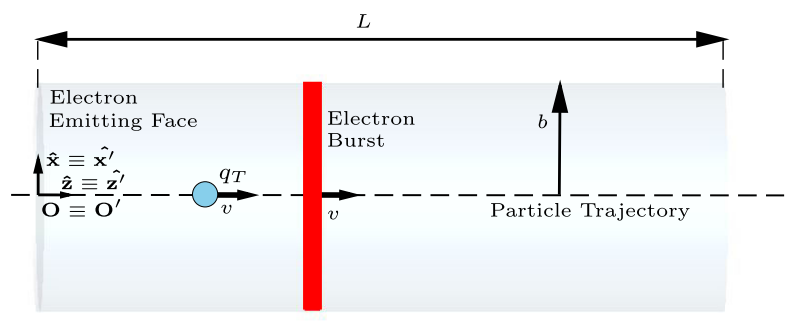

(a)

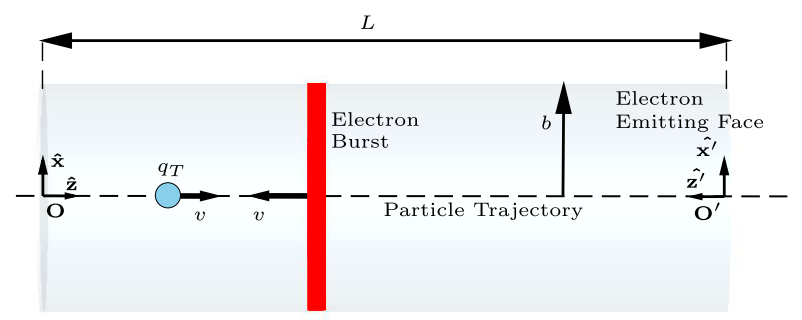

(b)

FIG. 8. Pillbox cavity excited by an electron burst emitted from one of the circular faces. It is important to stress that, for the countermoving case the reference frame in which the wake-function is needed, $\mathbf{O}$, and the reference frame in which Eq. (39) gives the electric field, $\mathbf{O}^{\prime}$, are not coincident. (a) Comoving case. (b) Countermoving case.

where, $N$ is the number of electrons per square meter, $J_{0}(x)$ is the Bessel function of order zero, $\rho_{m}$ are the roots of the Bessel function $J_{0}, I_{0}(x)$ is the modified Bessel function of zero order, $U(t)$ is the unit function, $\gamma_{S}=1-\beta_{S}^{2}, \gamma_{1}^{2}=$ $\rho_{m}^{2}+(n \pi a / L)^{2}$ and $\gamma_{2}^{2}=\rho_{m}^{2}+(n \pi a)^{2}\left(1-\beta_{S}^{2}\right) / L^{2}$. Furthermore, $r_{q_{T}}(t)^{\prime}$ and $z_{q_{T}}(t)^{\prime}$ are the generic radial and longitudinal positions of a test particle $T$ in a reference frame $\mathbf{O}^{\prime}$ which has origin in the electron emission face and $\hat{\mathbf{z}}^{\prime}$ is oriented in the direction of motion of the electrons. The electric field $E_{z}^{\prime}$ given by Eq. (39) is also expressed in the $\mathbf{O}^{\prime}$ reference frame. Finally, $t$ is the generic time.

This section aims at expressing the longitudinal wakefunction in a reference frame $\mathbf{O}$, for which the $z$ axis is directed in the test charge direction of motion and its origin is fixed at the test entrance section. For the comoving scenario $\mathbf{O}$ and $\mathbf{O}^{\prime}$ are coincident, however this is not true for the countermoving scenario. In the following the quantities with the superscript ' are considered to be expressed in $\mathbf{O}^{\prime}$ frame, the quantities without ' are considered to be expresses in the $\mathbf{O}$ frame.

In the comoving scenario, the reference frames $\mathbf{O}$ and $\mathbf{O}^{\prime}$ are coincident, thus, one can write [considering also Eq. (31)]:

$$
\begin{aligned}
r_{q_{T}}^{\prime} & =r_{q_{T}} \\
z_{q_{T}}^{\prime} & =z_{q_{T}}=v t-v \Delta t_{S T} \\
E_{z}^{\prime} & =E_{z} .
\end{aligned}
$$

Equations (40) are substituted into Eq. (39) to obtain the longitudinal electric field $E_{z}$. Subsequently, using Eq. (4), the longitudinal comoving wake-function can be obtained.

In the counterrotating wakefield case, the quantities in $\mathbf{O}^{\prime}$ are linked to the quantities in $\mathbf{O}$ by the following equations [considering also Eq. (32)]:

$$
\begin{aligned}
r_{q_{T}}^{\prime} & =r_{q_{T}} \\
z_{q_{T}}^{\prime} & =L-z_{q T}=L-v t+v \Delta t_{S T} \\
E_{z}^{\prime} & =-E_{z} .
\end{aligned}
$$

The first two equations can be intuitively derived looking at Fig. 8(b), where both reference frame $\mathbf{O}$ and $\mathbf{O}^{\prime}$ are represented. The third relation comes from the fact that the $z$ axes of the two frames point in opposite directions.

Substituting Eqs. (41) into Eq. (39) the longitudinal electric field is obtained in the $\mathbf{O}$ frame and the longitudinal countermoving wake-function can be computed using Eq. (4).

The co- and countermoving wake-functions were evaluated integrating numerically the longitudinal electric field $E_{z}$ for the case in which both the electrons and the test particle are ultrarelativistic, i.e., $\beta_{S}=1$ and $v=c$, and with the test particle travelling on the pillbox axis, i.e., $r_{q_{T}}^{\prime}=r_{q_{T}}=0$. The wake-functions are reported in Fig. 9 as a function of the entrance delays. Their Fourier transforms, the co- and countermoving impedance, in absolute values, are reported in Fig. 10.

From the wake-function, the wakepotential was numerically computed by convolution with a Gaussian distribution.

To benchmark the validity of the calculations, simulations of the wakepotential were performed. Using the particle in cell (PIC) solver of CST, the excitation of a lossless pillbox (length $L=0.6 \mathrm{~m}$ and radius $a=0.1 \mathrm{~m}$ )

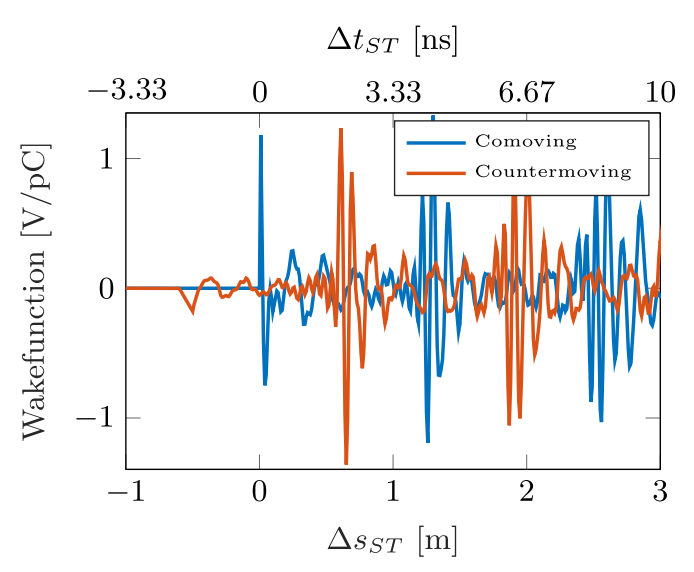

FIG. 9. Comparison between the comoving and the countermoving wake-function of a pillbox cavity excited by a burst of electrons emitted by one of the faces. The wake-functions have been obtained with the Faust theory. 


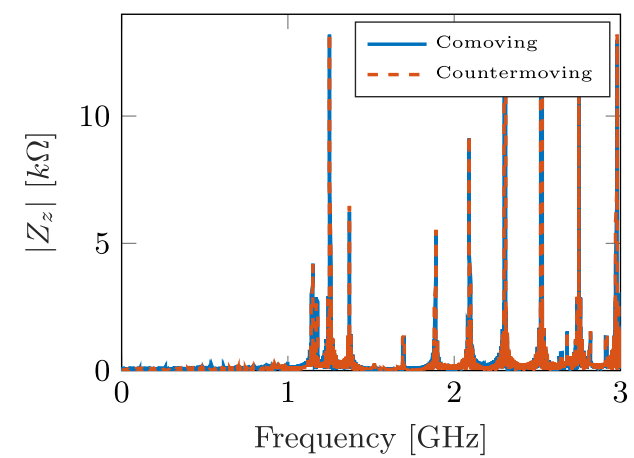

FIG. 10. Comparison between the comoving and the countermoving impedance of a pillbox cavity excited by a burst of electrons emitted by one of the faces.

by a burst of electrons emitted by one of the circular face was simulated. The cavity material was set to be a perfect electric conductor (PEC), so that the cavity was loss free. The electrons were emitted uniformly from the face with a Gaussian longitudinal distribution $\left(\sigma_{b}=0.07 \mathrm{~m}\right)$. One bunch of electrons with a total charge of $1 \mathrm{nC}$ $\left(6.24 \times 10^{9}\right.$ electrons $)$ was emitted. The kinetic energy of the electrons was set to an ultrarelativistic value $\left(\gamma=5 \times 10^{10}\right)$ to avoid space charge effects. Electric field monitors were set to register and store the value of the longitudinal electric field every $1.5 \mathrm{~mm}$ along the whole cavity axis, that is every $5 \times 10^{-3} \mathrm{~ns}$. The position of the test particle $T$ is known at every time $t$ as a function of the entrance delay $\left(z_{q_{T}}(t)=v t-\Delta s_{S T}=v t-v \Delta t_{S T}\right)$, i.e., fixing an entrance delay $\Delta s_{S T}$ or $\Delta t_{S T}$, one knows $T$ 's longitudinal position $z_{q_{T}}$ at the time $t$. If $z_{q_{T}}$ at the time $t$ is known, one can obtain the value of the longitudinal electric field acting on $T$ at the time $t$ from the fields monitors. If this operation is repeated for every $t$ one obtains the longitudinal electric field experienced by $T$ traversing the cavity as a function of time (or equivalently as a function of its longitudinal position). Integrating this longitudinal electric field gives the wake-potential value for the set entrance delay, and repeating the integration for different entrance delays gives the whole wake-potential.

The counterrotating wake-potentials as a function of the entrance delay between $Q_{S}$ and $T\left(\Delta s_{Q_{S} T}\right.$ and $\left.\Delta t_{Q_{S} T}\right)$ obtained from the formal model (indicated as semianalytic) and the CST PIC solver (indicated as CST) are reported and compared in Fig. 11(b). The agreement between the two methods is excellent.

\section{Discussion}

The model exposed in Sec. II is capable of reproducing known results from the literature, i.e., the comoving wakepotential of a resistive wall pipe [as shown in Fig. 7(a)]. Additionally, its results compare well with simulations of simple geometries, i.e., the comoving wake-potentials of a resistive wall pipe and a lossless pillbox cavity [Figs. 7(a) and 11(a)].

Furthermore, the model provides a tool to estimate the results of the interaction of the countermoving beams, i.e., countermoving wake-functions (Figs. 6 and 9) and wakepotentials [refer to Figs. 7(b) and 11(b)].

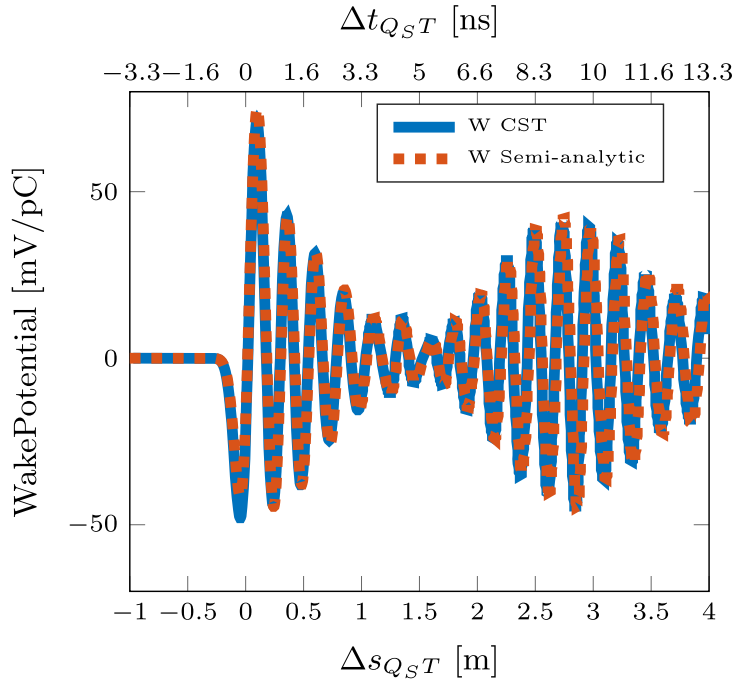

(a)

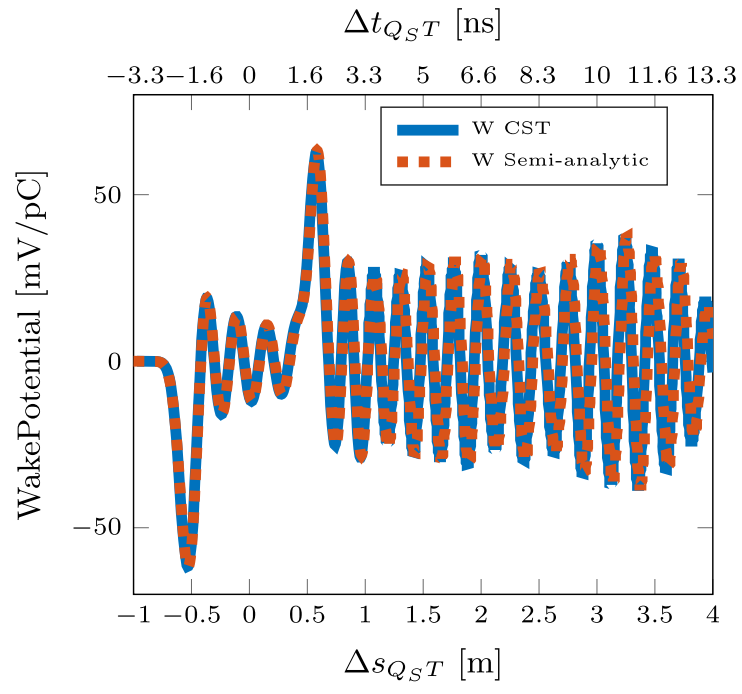

(b)

FIG. 11. Comoving (a) and countermoving (b) wake-potentials of a burst of ultrarelativistic electrons $\left(1 \mathrm{nC}\right.$ or $6.24 \times 10^{9}$ electrons and $\beta_{S} \rightarrow 1$ or $\gamma_{S}=5 \times 10^{10}$ ) traversing a pill box cavity (length $L=0.6 \mathrm{~m}$ and radius $a=0.1 \mathrm{~m}$ ) computed by the proposed model (using the Faust theory [23]) and the CST PIC solver [24]. The electrons are emitted uniformly from one of the circular faces of the cavity. Their longitudinal distribution is a Gaussian bunch $\left(\sigma_{b}=0.07 \mathrm{~m}\right)$. The cavity material is a perfect electric conductor (PEC), loss free. Test particle speed is $v=c$. (a) Comoving Wakepotential. (b) Countermoving Wakepotential. 
Finally, the countermoving wake-potentials are in good agreement with numerical simulations results [Figs. 7(b) and 11(b)].

The last consideration is important. It implies that, not only the presented theoretical model is able to describe the countermoving wakefield effects, but it also served as a check of CST Studio Suite (version 2018, Wakefield and PIC solvers) results supporting the use of the code in more realistic and complex geometries.

There are also other points to discuss, relating to the results of the proposed formalism in the two shown examples.

For both the resistive wall pipe and the lossless pillbox cavity, the comparison between comoving and countermoving functions shows that these two quantities are different, and apparently, there is no trivial relation between them. This is especially true for the transient region of the wakefield, i.e., the interval in the entrance delay for which the two beams are both transiting inside the vacuum chamber.

For the resistive pipe, the countermoving wake-function is reported in Fig. 6. Remarkably, the wake-function is characterized by two peaks, one positive and one negative, symmetrically arranged with respect to the axis origin with space entrance delay equal to the length of the pipe. These peaks are due to boundary conditions at the end and at the beginning of the pipe. Indeed, Eq. (33), that holds for an indefinitely long pipe, has been used to compute the wakefunction of a finite length pipe, considered as a segment of this infinitely long pipe. In the longitudinal wake-function definition, Eq. (4), it is assumed that outside of the structure, the electric fields can be neglected. This cut is also performed by the CST software when a pipe of length $L$ is simulated. The software is able to compute only the fields inside the structure and it assumes null the field outside. This means that both in the simulations and the model the boundary conditions at the extremities are considered in the same way, thus explaining the good agreement in the countermoving wake-potentials shown in Fig. 7(b).

Regarding the pillbox cavity, a comparison between its comoving and countermoving wake-function is reported in Fig. 9. As one can see, the wake-functions present some differences, especially in the transient region around 0 entrance delay, for $\Delta s_{S T}<L$, with $L=0.6 \mathrm{~m}$ as the length of the cavity. While for $\Delta s_{S T}>L$ they seem to be similar but translated. This can be explained by the fact that the effects of the countermoving wakefields differ the most from the comoving ones when the source and the test meet inside the component they are transiting. If the test enters into a pillbox cavity that has already been excited by a source particle and if this source particle has already escaped from the component, in principle the test particle should not be able to distinguish if the source particle was comoving or countermoving. This also means that, the greater the cavity or beam length, the longer the transient and the larger the differences between co and countermoving wakefields effects.

With reference to Fig. 10, the comoving and countermoving impedance modulus compare very well. In particular the resonant mode frequencies shown by the comoving impedance are the same as those shown by the countermoving impedance. This result was expected since the resonant frequency of a mode is a property of the device, and it is independent of the excitation source.

\section{B. Study of the energy dissipated by a two beam system transiting a lossy pillbox cavity}

In this subsection the energy losses of two countermoving beams traversing a resonant cavity are discussed as a function of the entrance delay between the beams.

The subsection aims at benchmarking Eqs. (21)-(26) for computing the energy variations of a system of two countermoving beams. Furthermore the real and imaginary parts of the comoving and countermoving impedance of the cavity are compared.

The subsection is divided in two parts, in the first part the methodology to obtain longitudinal comoving and countermoving impedance, and the related energy variation is described and all the results are presented. In the second part the physical meaning of the results is discussed.

\section{Methodology and results}

Using the CST 2018 Wakefield solver [24] the passage of two countermoving beams, both positioned exactly at the center of the structure, was modeled for the pillbox cavity shown in Fig. 12. Both beams were composed of a single Gaussian bunch with a charge $q=4.5 \times 10^{-8} \mathrm{C}$ and a root mean square bunch length $\sigma_{b}=70 \mathrm{~mm}$. One of the beams was simulated entering the structure at the time $t_{1 i}=0$. This beam is labeled as beam one in the following. The other beam (beam two) was simulated entering the structure with an arbitrary delay $\Delta t_{b_{1} b_{2}}$ with respect to beam one.

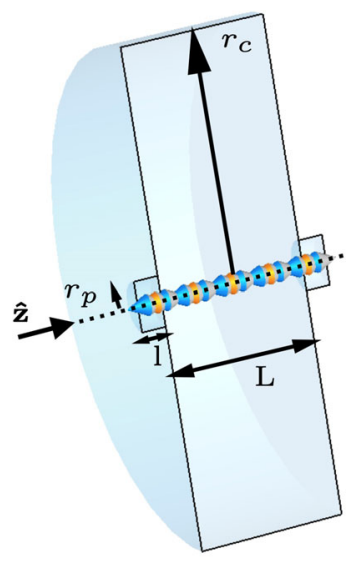

FIG. 12. Simulated cavity model with geometrical quantities: $r_{c}=100 \mathrm{~mm}, r_{p}=10 \mathrm{~mm}, L=60 \mathrm{~mm}, l=10 \mathrm{~mm}$. 
The pillbox cavity shown in Fig. 12 was built such that the beams were able to excite only the first longitudinal resonant mode, a $\mathrm{TM}_{010}$ mode $[25,26]$. Furthermore, the electrical conductivity of the cavity wall was set to $\sigma=500 \mathrm{~S} \mathrm{~m}^{-1}$. This low value was used to force a quick damping of the fields of the excited mode and to speed up simulation time.

The CST Wakefield solver returns the wake loss factor for each one of the two beams. Inverting Eqs. (20), the beam loss factor can be used to obtain the energy variation of the considered beam in transiting the device. Summing the energy variation of the two beams, the total energy loss of the two beam system while transiting the chamber is computed. For each investigated value of $\Delta t_{b 1 b 2}$ one simulation is required. In the following, the total energy loss obtained directly by post-processing the CST loss factors will be labeled as $\Delta U_{\mathrm{CST}}$.

In checking the results of the CST code, the authors found an issue. The CST 2018 Wakefield solver [24], used for simulations, computed automatically the loss factor of a beam. However, due to a problem in the software, when two beams were simulated, for each, the loss factor of the last defined beam was shown. Thus, to obtain the correct value of the loss factor for the two beams, for each considered $\Delta t_{b 1 b 2}$, two simulations had to be repeated defining the two beams in different order. A ticket with the software support team was opened and the issue was fixed in the CST 2021 Wakefield solver.

The energy variations of the two beams as a function of the entrance delay between them $\Delta t_{b 1 b 2}$ were obtained also using Eqs. (21)-(23). To obtain the countermoving impedances terms $\left(Z_{12}, Z_{21}\right)$ and the comoving ones $\left(Z_{11}, Z_{22}\right)$, that are needed in Eqs. (21)-(23), two CST simulations were run. In each simulation, a source beam with charge $q_{S}=4.5 \times 10^{-8} \mathrm{C}$, composed of a single Gaussian bunch $\left(\sigma_{b}=70 \mathrm{~mm}\right)$ was simulated directed along the $\hat{\mathbf{z}}$ axis (with reference to Fig. 12). Additionally to the source beam, two test beams, with the same characteristics, and with a total charge of $q_{T}=4.5 \times 10^{-11} \mathrm{C}$ were simulated, one directed in the positive $\hat{\mathbf{z}}$ axis $(+\hat{\mathbf{z}})$ and the other directed in the negative $\hat{\mathbf{z}}$ axis $(-\hat{\mathbf{z}})$. Since the test charges are negligible with respect to the source beam charge, one can reasonably make the approximation that the electromagnetic fields in the cavity are due only to the source beam. In a first simulation the source beam was directed along the positive $\hat{\mathbf{z}}$ axis. The impedance of the test beam directed along $+\hat{\mathbf{z}}$ has to be scaled by a factor $q_{T} / q_{S}$ to obtain $Z_{11}$. On the other hand, the impedance of the test beam in the $-\hat{\mathbf{z}}$ direction has to be scaled by the same factor $q_{T} / q_{S}$ to obtain $Z_{12}$. The scaling factor $q_{T} / q_{S}$ comes from the fact that the source and test beams do not have the same charge. In the second simulation, the source beam was directed along the negative $\hat{\mathbf{z}}$ axis. Thus, the impedances of the test beams directed along $+\hat{\mathbf{z}}$ and along $-\hat{\mathbf{z}}$ had to be scaled by $q_{T} / q_{S}$ to obtain $Z_{12}$ and $Z_{22}$, respectively.
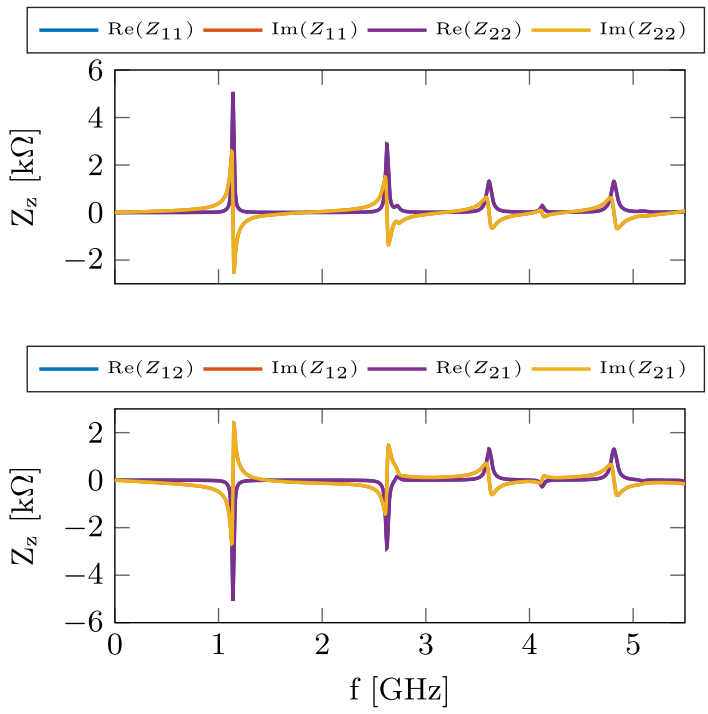

FIG. 13. Simulated comoving impedances $\left(Z_{11}, Z_{22}\right)$, top, and countermoving impedances $\left(Z_{12}, Z_{21}\right)$, bottom, for the pillbox cavity shown in Fig. 12. Some curves are not visible because they completely overlap with others. The impedance curves in this figure were obtained simulating a Gaussian bunch with $\sigma_{b}=20 \mathrm{~mm}$ to excite frequencies up to $5 \mathrm{GHz}$. In the rest of the analysis presented in this section the value $\sigma_{b}=70 \mathrm{~mm}$ was used.

The real and imaginary parts of the impedances are reported in Fig. 13.

Using the obtained values of comoving and countermoving impedances, and substituting them into Eq. (21), one obtains the total energy dissipated by the two beam system in the pillbox as a function of the beam entrance delay in the device.

In Fig. 14, a comparison between the total energy lost by the two beam system computed using Eq. (21) and by CST is reported. The results agree very well.

In Fig. 15 the energy variation for each single beam and the total energy losses of the two beams, computed using Eqs. (21)-(23) as a function of the entrance delay between the two beams, are reported. They are normalized by a reference energy variation $\Delta U_{r}$, that is the energy that one beam would lose transiting alone into the cavity.

Furthermore, given the symmetry of the simulated cavity, Eq. (28) was used to find the values of the entrance delay between the beams at which the maxima and the minima of the total energy losses were corresponding. Since the beams mainly exchange power with the resonant cavity via the excited mode, and since the beams can only excite the mode that resonates at a frequency $f_{r}=1.1418 \mathrm{GHz}$, the power exchange happen mainly at that frequency. Thus, $f_{r}=1.1418$ was the frequency value used in Eq. (28). Considering different values for $n$, the values of $\Delta t_{b_{1} b_{2}}$ that maximise or minimize the energy lost by the beams were found. The energy dissipated in the pillbox cavity as a function of $\Delta t_{b_{1} b_{2}}$ is reported in Fig. 16 


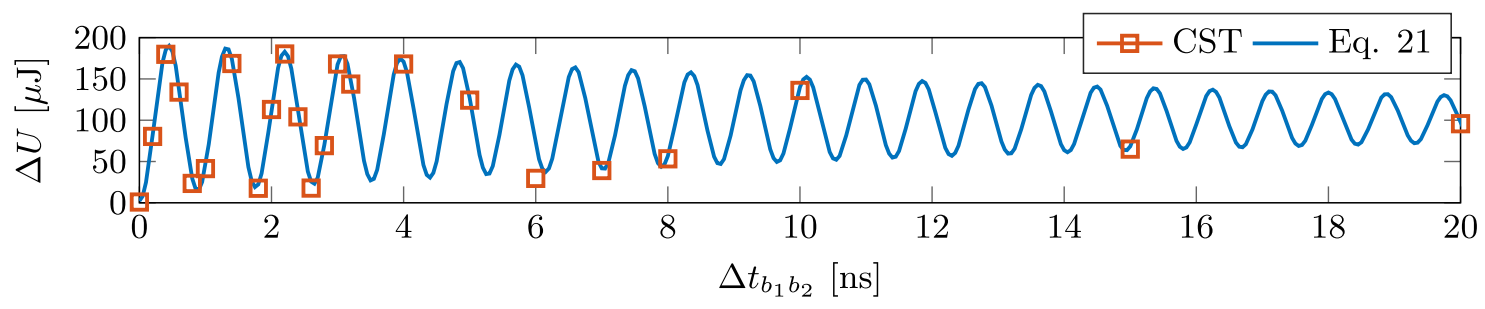

FIG. 14. Comparison between the total energy variation of the two beams system obtained directly from CST and from Eq. (21). To obtain a single value of the total energy variation from CST, two simulations had to be performed. Since these simulations were time consuming, the resolution of the $\Delta U$ points obtained with CST was decreased moving from low to high values of the entrance delay.

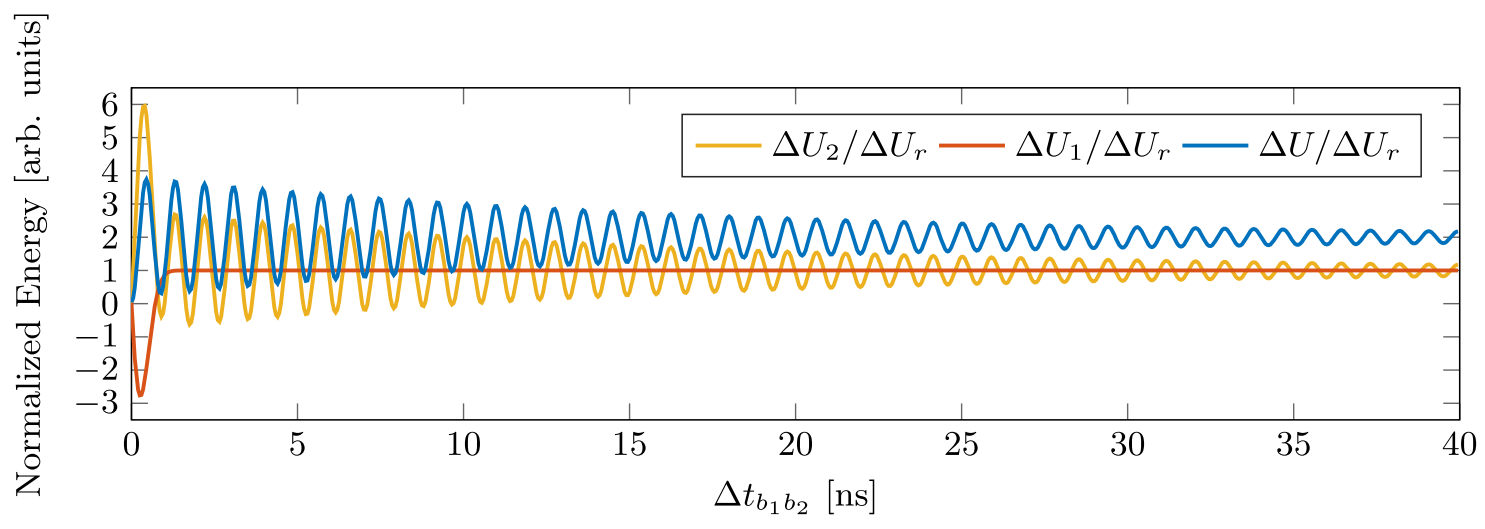

FIG. 15. Normalized total energy losses and energy variation of beam 1 (the first beam to enter into the cavity) and beam 2 (the delayed beam) as a function of the entrance delay between the two beams $\left(\Delta t_{b_{1} b_{2}}\right)$, for the cavity reported in Fig. 12.

where also the coordinate of the maxima and minima obtained by Eq. (28) are highlighted.

\section{Discussion}

Regarding the impedances shown in Fig. 13, one can notice immediately that $Z_{11}=Z_{22}$ and that $Z_{12}=Z_{21}$.

These relations were expected to hold because the geometry of the problem is longitudinally and transversely symmetric.

However, they are valid also under other conditions. If the orbits of the two beams traversing the vacuum chamber are superimposed, as in the proposed example, the relation

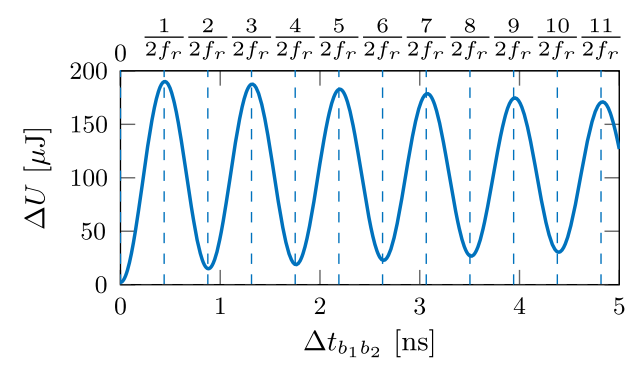

FIG. 16. The energy dissipated in the pillbox cavity traversed by two beams is reported, as a function of the entrance delay between the beams, $\Delta t_{b_{1} b_{2}}$. The values of $\Delta t_{b_{1} b_{2}}$ that correspond to the maxima and minima of the energy losses, obtained by Eq. (28), are highlighted.
$Z_{11}=Z_{22}$ is a consequence of the reciprocity theorem [27] and it is valid even if the vacuum chamber has no symmetries.

Furthermore, preliminary simulation results show that, for longitudinally symmetric devices, the relations $Z_{12}=Z_{21}$ holds also if there is no transverse symmetry. Contrarily, the relations $Z_{12}=Z_{21}$ seems not to be verified when devices without longitudinal symmetry were simulated. Detailed studies on the symmetry proprieties of the countermoving impedance go beyond the scope of this paper and will be the subject of future work.

Returning to the results shown in Fig. 13, both comoving and countermoving impedances show the resonant modes at the same frequencies, as in the example discussed in Sec. III A 2. Furthermore, for the countermoving impedance, some modes show positive real part impedance, others negative.

Close to the resonances, the ratio between comoving and countermoving shunt impedances assumes always the values -1 or 1 . Thus, the shunt impedance of the mode seen by the comoving beam and the shunt impedance of the mode seen by the countermoving beam have the same absolute value.

This relations between comoving and countermoving impedances for longitudinally symmetric objects was already predicted by the Wang model [1]. For longitudinal impedances, this behavior may be explained considering 
that the countermoving impedances, $Z_{12}$ and $Z_{21}$, take into account the energy exchanged by the beams via the resonant mode. Thus, a negative real part indicates that one of the beams is gaining energy from the resonant mode that was excited at the expense of the other beam, which provided the energy. A positive real part indicates that one of the beams is losing energy during its interaction with the mode that has been excited by the other beam.

Regarding the energies, the total energy dissipation of the two beam system obtained numerically and by Eq. (21) agree well (as shown in Fig. 14). This is another benchmark for both the CST software and the proposed formula.

Figure 14 clearly shows a damped oscillating behavior of the total energy lost by the two beam system as a function of the entrance delay between the beams. The physical reasons of such a behavior can be better understood by considering how the countermoving beams interact with the $\mathrm{TM}_{010}$ mode.

A $\mathrm{TM}_{010}$ in a pillbox cavity is characterized by an electric field parallel to the axis of the pillbox $(\hat{\mathbf{z}})$, and its local magnitude at an arbitrary time is independent of the longitudinal coordinate $(z)$.

Each of the two beams entering the cavity excites the $\mathrm{TM}_{010}$ mode fields independently. The total field in the cavity is the sum of the fields excited by each beam. This leads to an interference-like behavior of the total field: depending on the entrance delay between the two beams $\Delta t_{b_{1} b_{2}}$, there could be constructive or destructive interference. As an example, one can consider the case for which the entrance delay between the two identical countermoving beams is null. In this case, because of the field pattern of the $\mathrm{TM}_{010}$ mode and because of the counterrotation, the electromagnetic fields excited by one beam have the same intensity and opposite direction of those excited by the other beam, i.e., the fields are excited with an opposite phase. This gives a null total field. Following this reasoning, if a beam enters into the cavity with a delay with respect to the other one of half of the time period of the $\mathrm{TM}_{010}$ resonant mode, i.e., $\Delta t_{b_{1} b_{2}}=1 /\left(2 f_{r}\right)$, with $f_{r}$ the mode resonant frequency, there is a constructive interference. Indeed, the fields excited by the delayed beam (beam two) when entering the cavity have the same instantaneous intensity and direction of the fields excited by the previous beam (beam one). Thus, they produce a constructive interference. If beam two enters with a delay that is equal to the mode resonant period, i.e., $\Delta t_{b_{1} b_{2}}=1 / f_{r}$, the mode fields excited by each beam are again in phase opposition and there is again a destructive interference.

The energy to excite the total electromagnetic fields in the pillbox is provided by the whole two beam system. Thus, one expects that values of $\Delta t_{b_{1} b_{2}}$ that lead to constructive interferences correspond to high energy losses These reasoning is coherent with the results shown in Fig. 16. In this figure, the total energy lost by the two beams is shown and the time entrance delays that corresponds to the energy loss maxima and minima, found with Eq. (28), are highlighted. As expected, for a null value of the beam entrance delay the total dissipated energy has a minimum. Furthermore, the other energy maxima and minima are located, according to Eq. (28), at half of the time period of the resonant mode.

It is important to stress that the interference behavior, in general, depends on the field pattern and frequency of the mode, as well as the entrance delay between the two beams. This may be a key aspect to consider for collision chamber, in which, usually, the entrance delay between the beams is null.

Additional information on the interaction of the countermoving beams among themselves and with the cavity mode can be understood if one looks at the normalized energy variations of the single beams that are reported in Fig. 15. As anticipated, if the entrance delay between the two beams is null, they both excite the mode inside the pillbox cavity but with an opposite phase. The net result is that the mode inside the cavity is not excited and so the only dissipated energy is due to the resistive wall effects. If the delay between the beams is different from zero but such that the two beams meet each other inside the pillbox cavity (transient time interval) there is a more complex scenario. The first beam that entered (beam one) gains energy while the delayed beam loses energy. The energy lost by beam two is higher than the energy gained by beam one, the extra energy is going to the vacuum chamber as heat. If the entrance delay is increased, beam one no longer meets beam two inside the pillbox cavity because the delay is such that beam one has left the cavity before beam two enters. This explains the fact that after about $1.5 \mathrm{~ns}$ $\Delta U_{1} / \Delta U_{r}$ stabilizes around the value of 1, i.e., the value of the energy loss that one beam would have if it had crossed the vacuum chamber alone. In the case in which beam two enters in the cavity after the exit of beam one, it finds a resonant mode that is already excited in the cavity. Beam two interacts with the resonating mode, depending on the entrance delay it can absorb energy from the mode or excite it more, as it is visible from the oscillations of $\Delta U_{2} / \Delta U_{r}$. The oscillation is damped by the fact that the mode in the cavity dissipates energy heating the cavity walls. Thus, the higher $\Delta t_{b_{1} b_{2}}$ is, the lower the energy available for exchanges with the beam is. As expected the damped oscillations converge toward the value of 1 for $\Delta t_{b_{1} b_{2}} \rightarrow \infty$. Indeed, if the beam entrance delay is large enough that the mode excited by the first beam is completely decayed when the second beam arrives, the latter is in the same situation of the first one: it arrives finding an empty cavity and with its passage it excites the mode. The normalized total energy variation of the two beams $\Delta U / \Delta U_{r}$ has an oscillating behavior and it is always positive: as energy conservation law dictates for the system, energy is always dissipated in the cavity wall. Its oscillations have a maximum of about 4 , this means that 
in the worst case scenario, two countermoving beams can dissipate on a resonant mode up to four times the energy that would be dissipated by only one beam, when their fields constructively add up in phases. This finding is in agreement with the prediction of the models of Grudiev [15] and Zannini et al. [13] and with the observation of Giordano et al. [16]. On the other hand, for specific values of the beams entrance delay $\Delta t_{b_{1} b_{2}}$, the total energy dissipated on the cavity approaches the zero value.

Finally, as shown in Fig. 16, the presented Eq. (28) was able to identify maxima and minima of the total energy lost by the two beams system.

This last result may be crucial during the design phase of a device traversed by two beams. In standard operations of a collider, the time entrance delay between the two beams is fixed. For instance in a longitudinally symmetric interaction chamber the beams have to enter with $\Delta t_{b_{1} b_{2}}=0$ to properly collide, while in another component the entrance delay depends only from its position with respect to the collision points in the accelerator. Thus, Eqs. (27)-(28) could be used during the design phase of a device to understand if, for that entrance delay, the beams will interact with the device modes in a constructive or destructive way. The former case can be dangerous because the device can experience large energy deposition. If this happens, the device geometry or its materials should be modified to move the resonant frequency of the dangerous modes. Also repositioning the device to a different accelerator location could help. Indeed this would change the beam entrance delay $\Delta t_{b_{1} b_{2}}$, so avoiding the constructive resonance of modes.

\section{CONCLUSIONS AND FUTURE WORK}

This paper has introduced a model to describe both the comoving and the countermoving wakefield effects via the introduction of the concepts of space and time entrance delay. Furthermore, it has presented a way to simulate the longitudinal countermoving wakefield effects and it has successfully benchmarked the proposed model against simulations data from the commercial software CST Studio Suite 2018. The benchmark allowed the authors to check that both the model and the CST software can estimate the longitudinal countermoving beams effects.

The reported results have shown that the model could retrieve literature results for the comoving beams and could estimate the longitudinal wake-function (and so the impedance) and the longitudinal wake-potential of two countermoving beams for two common geometries. In the computation for the countermoving wakefields case, the effects of the beams on each other at the transient time in which they are both transiting inside the same vacuum chamber has been taken into account. Comparing the obtained comoving and countermoving wake-functions and wake-potentials for the same geometry it was shown that there are differences. The behavior of comoving and countermoving wake-potential is involved and the largest differences are concentrated on the timespan in which the beams transit simultaneously inside the device.

In addition, the expression of the energy and the power dissipated in a device by two countermoving beams was given. As expected from other literature results, it was shown that the beams interact with a mode with an interference like behavior. The energy (or power) dissipated in a device by two countermoving beams can vary from 0 to 4 times the energy dissipated by a single beam, depending on the entrance delay of the two beams into the vacuum chamber. Using the physical model developed in the paper it was also possible to localize the beam entrance delay that gives the maxima and the minima of the dissipated energy and power. It was discussed how this can be a crucial parameter in the early design phase of the device.

Future work should study the symmetry proprieties of the countermoving wake-functions and impedances and should investigate the physical reasons behind them. It should also benchmark Eq. (27) to estimate the dissipated power maxima and minima for a longitudinally nonsymmetric object and the case in which several modes are excited at the same time.

Finally, the model proposed in this paper could be used to further investigate the effects on the beam dynamics of the longitudinal and the transverse countermoving wakefield.

\section{ACKNOWLEDGEMENT}

The authors would like to thank F. Zimmerman who provided Refs. [1,10] and [12]. This work was partly supported by the European commission under HORIZON 2020 integrating activity project ARIES, Grant agreement No 730871 and by INFN national committee through the ARYA project.

\section{APPENDIX: BENCHMARK OF THE PANOFSKY-WENZEL THEOREM IN SIMULATIONS FOR TWO COUNTERMOVING BEAMS}

In this Appendix the Panofsky-Wenzel theorem is verified in the two beams scenario by using CST. This is a further benchmark for the software.

The simulated geometry is the resonant cavity reported in Fig. 17, which includes also the in/out pipes (only one is visible in the image). The reference system $\mathbf{O}$ is also represented in the left hand side of the figure: the origin is at the center of the pipe and $\hat{\mathbf{z}}$ defines the longitudinal direction.

Two countermoving bunches (bunch one and bunch two) with Gaussian shape, with the same bunch length, $\sigma_{b}=$ $15 \mathrm{~mm}$ and the same total charge, $q=1 \mathrm{nC}$, were simulated. For each bunch, the trajectory of the source charges (the trajectory of the excitation source) and of the test 


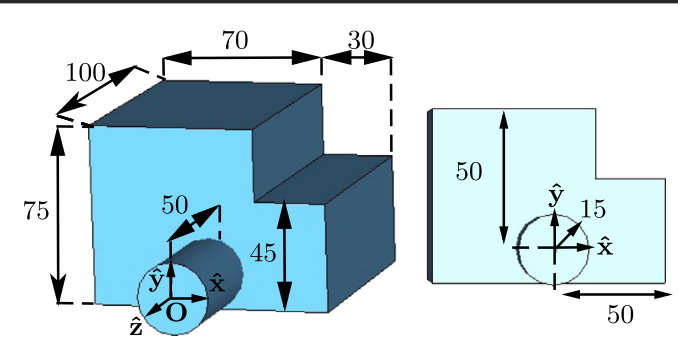

FIG. 17. Simulated cavity for the benchmark of the PanofskyWenzel theorem. The dimensions are in millimiters.

charges (the trajectory where the impedance is calculated) had to be defined. These trajectories were set parallel to the $\hat{\mathbf{z}}$ axis. Thus, they are identified only by their transverse coordinates, i.e., $x_{S b_{1}}, y_{S b_{1}}, x_{S b_{2}}, y_{S b_{2}}$ for the source trajectories, and $x_{T b_{1}}, y_{T b_{1}}, x_{T b_{2}}, y_{T b_{2}}$ for the test trajectories. The subscripts $b_{1}$ and $b_{2}$ refer to bunch one and bunch two, respectively. For the sake of simplicity, the source trajectories of bunch one and bunch two were considered coincident and the same was done for the test trajectories. Thus, the following equations hold:

$$
\begin{aligned}
& x_{S b_{1}}=x_{S b_{2}}=x_{S} \\
& y_{S b_{1}}=y_{S b_{2}}=y_{S} \\
& x_{T b_{1}}=x_{T b_{2}}=x_{T} \\
& y_{T b_{1}}=y_{T b_{2}}=y_{T}
\end{aligned}
$$

An entrance delay $\Delta t_{b_{1} b_{2}}=0$ between the bunches was considered, i.e., the bunches entered into the cavity at the same time.

The CST software returns the impedances of the cavity excited by the counterrotating bunches as a function of frequency for the three axes: $Z_{x}, Z_{y}$ and $Z_{z}$.

According to the Panofsky-Wenzel theorem in its impedance form [28], the following relations have to hold:
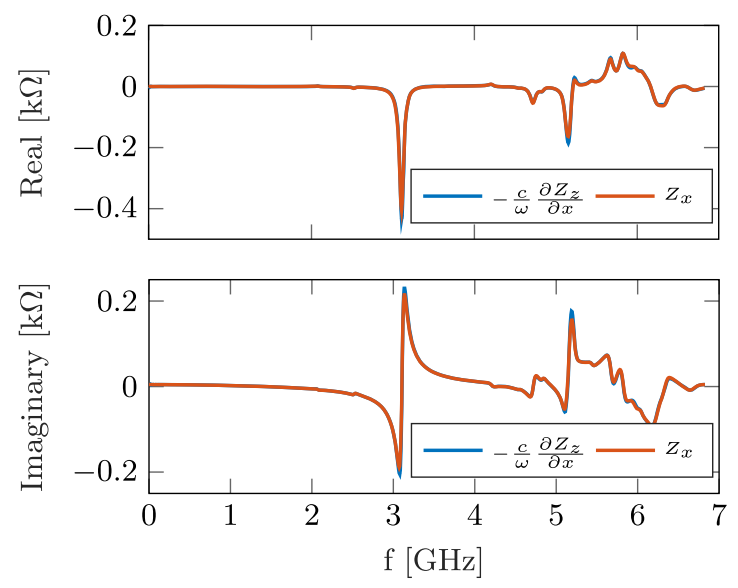

$$
\begin{aligned}
& Z_{x}\left(x_{S}, y_{S}, x_{T}, y_{T}\right)=-\frac{c}{\omega} \frac{\partial Z_{z}\left(x_{S}, y_{S}, x_{T}, y_{T}\right)}{\partial x_{S}}, \\
& Z_{y}\left(x_{S}, y_{S}, x_{T}, y_{T}\right)=-\frac{c}{\omega} \frac{\partial Z_{z}\left(x_{S}, y_{S}, x_{T}, y_{T}\right)}{\partial y_{S}} .
\end{aligned}
$$

In Eqs. (A2), the dependence of $Z_{x}, Z_{y}$, and $Z_{z}$ on the test and source trajectories $\left(x_{S}, y_{S}, x_{T}, y_{T}\right)$ have been explicitly indicated, $c$ is the speed of light and $\omega=2 \pi f$, where $f$ is the general frequency.

To verify Eqs. (A2) five simulations were required. For all the simulations the impedance was computed setting the test trajectory position at the value $x_{T}=y_{T}=0$, i.e., along the in/out pipe axis.

One simulations was done considering source and test trajectories coincident, i.e., $x_{S}=y_{S}=0$, this provided the terms $Z_{x}, Z_{y}$ of Eqs. (A2).

Subsequently, to compute $\partial Z_{z} / \partial x_{S}$ the source trajectory was moved around the test trajectory along the $\hat{\mathbf{x}}$ axis, in the positions $x_{S_{0}}=-1 \mathrm{~mm}$ and $x_{S_{1}}=1 \mathrm{~mm}$, keeping $y_{S}=0$. In both positions the longitudinal impedance was computed at $x_{T}=y_{T}=0$ and its derivative with respect to $x$ was obtained using the finite difference formula:

$$
\frac{\partial Z_{z}}{\partial x_{S}}=\frac{Z_{z}\left(x_{S_{1}}\right)-Z_{z}\left(x_{S_{0}}\right)}{x_{S_{1}}-x_{S_{0}}}
$$

To compute $\partial Z_{z} / \partial y_{S}$ the source trajectory was moved around the test trajectory along the $\hat{\mathbf{y}}$ axis, in the positions $y_{S_{0}}=-1 \mathrm{~mm}$ and $y_{S_{1}}=1 \mathrm{~mm}$, keeping $x_{S}=0$. In both positions the longitudinal impedance was computed at $x_{T}=y_{T}=0$ and its derivative with respect to $y$ was obtained using the finite difference formula:

$$
\frac{\partial Z_{z}}{\partial y_{S}}=\frac{Z_{z}\left(y_{S_{1}}\right)-Z_{z}\left(y_{S_{0}}\right)}{y_{S_{1}}-y_{S_{0}}}
$$

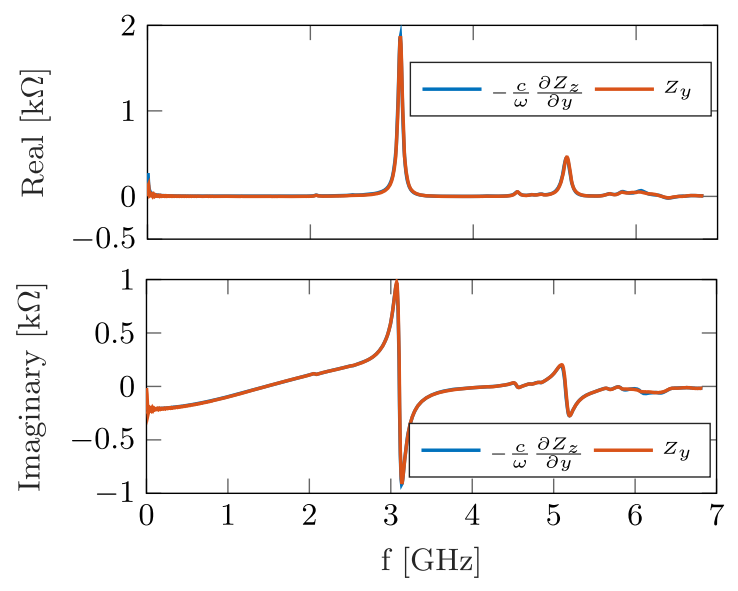

FIG. 18. Benchmark of the Panofsky-Wenzel theorem in a CST simulation. The simulated geometry is the cavity shown in Fig. 17, traversed by two countermoving bunches with delay $\Delta t_{b_{1} b_{2}}=0$. Some curves are not visible because they completely overlap with others. 
Once the terms $Z_{x}, Z_{y}, \partial Z_{z} / \partial x$, and $\partial Z_{z} / \partial y$ were computed, Eqs. (A2) could be verified. In Fig. 18 the real and imaginary parts of the right and the left terms of Eqs. (A2) are plotted as a function of frequency, showing an excellent agreement. Thus, this analysis shows that the CST software results in the countermoving beams' case satisfy the Panofsky-Wenzel theorem.

[1] J. Wang, Transverse two-beam instability, Report No. CERN/LEP-TH/87-65, 1987, https://cds.cern.ch/ record/183705.

[2] A. W. Chao, Physics of Collective Beam Instabilities in High Energy Accelerators (Wiley-VCH, New York, 1993), https://www.slac.stanford.edu/ achao/wileybook.html.

[3] K. Ng, Physiscs of Intensity Dependent Beam Instabilities (World Scientific, Singapore, 2002), https://doi.org/ $10.1142 / 5835$.

[4] B. W. Zotter and S. Kheifets, Impedances and Wakes in High Energy Particle Accelerators (World Scientific, Singapore, 1998), https://doi.org/10.1142/3068.

[5] K. L. F. Bane, P. B. Wilson, and T. Weiland, Wake fields and wake field acceleration, AIP Conf. Proc. 127, 875 (1985).

[6] O. S. Bruning, P. Collier, P. Lebrun, S. Myers, R. Ostojic, J. Poole, and P. Proudlock, LHC Design Report, CERN Yellow Reports: Monographs (CERN, Geneva, 2004), https://doi.org/10.5170/CERN-2004-003-V-1.

[7] D. C. Perez, N. Biancacci, C. Bracco, G. Bregliozzi, M. Calviani, M. I. Frankl, I. G. Lamas, L. Gentini, S. Gilardoni, G. Iadarola, A. Lechner, A. P. Marcone, and B. Salvant, Operational feedback and analysis of current and future designs of the injection protection absorbers in the Large Hadron Collider At CERN, in Proceedings of the 8th International Particle Accelerator Conference (IPAC 2017): Copenhagen, Denmark, May 14-19 (JACoW, Geneva, 2017), http://jacow.org/ipac2017/papers/wepva108 .pdf.

[8] L. Teofili, M. Migliorati, D. Carbajo, I. Lamas, and A. Perillo, Design of the Target Dump Injection Segmented (TDIS) in the Framework of the High Luminosity Large Hadron Collider (HL-LHC) Project, in Proceedings of the 61st ICFA Advanced Beam Dynamics Workshop on HighIntensity and High-Brightness Hadron Beams, Daejeon, Korea (JACoW, Geneva, 2018), pp. 122-126, https:// doi.org/10.1088/1742-6596/1067/6/062011.

[9] B. Salvant et al., Beam induced heating In LHC 2015, Report No. CERN-ACC-2016-279, 2016, http://cds.cern .ch/record/2207346? ln=en.

[10] C. Pellegrini, Longitudinal coupled bunch instability for two counterrotating beams, Report No. CERN-LEP-TH86-17, 1986, http://cds.cern.ch/record/170396.

[11] S. Myers, The LEP Collider, from design to approval and commissioning, Report No. CERN-91-08, 1991, https:// doi.org/10.5170/CERN-1991-008.
[12] F. Zimmermann, Two-beam resistive-wall wake field, in Proceedings of PAC07, Albuquerque, New Mexico, USA (IEEE, New York, 2007), pp. 4237-4239, https://cds.cern .ch/record/1056961/.

[13] C. Zannini, G. Rumolo, and G. Iadarola, Power loss calculation in separated and common beam chambers of the LHC, Report No. CERN-ACC-2014-0122, 2014, https://cds.cern.ch/record/1742192.

[14] G. Rumolo and C. Zannini, 2-beam impedance: review of theory, in Presentation Given at the CERN Impedance Meeting 29/03/2019, also available as https://indico.cern .ch/event/809558.

[15] A. Grudiev, Simulation and reduction of longitudinal and transverse impedances of a collimation device with two beams in one vacuum chamber, Report No. LHC-Projectnote-413, 2008, http://cds.cern.ch/record/1098139?1n=en.

[16] F. Giordano, G. Rumolo, B. Salvant, L. Teofili, and C. Zannini, Analysis of the 2 beam power loss in a cavity structure, in Presentation Given At The CERN Impedance Meeting, 20/10/2019, also available as https://indico.cern .ch/event/849584/.

[17] W. Herr and T. Pieloni, Beam-beam effects, Report No. CERN-2014-009, 2014, https://doi.org/10.5170/ CERN-2014-009.431.

[18] G. Rumolo, Beam instabilities, Report No. CERN-2014009, 2014, https://doi.org/10.5170/CERN-2014-009.199.

[19] CST Studio Suite, CST Studio Suite Website, also available as https://www.3ds.com/products-services/simulia/ products/cst-studio-suite/.

[20] W. A. Panofsky and W. K. H. Wenzel, Some considerations concerning the transverse deflection of charged particles in radio-frequency fields, Rev. Sci. Instrum. 27, 967 (1956).

[21] K. L. F. Bane, The short range resistive wall wakefields, Report No. SLAC/AP-87, 1991, https://www.slac.stanford .edu/pubs/apnotes/slac-ap-087.pdf.

[22] A. Piwinski, Longitudinal and transverse wakefield in flat vacuum chambers, Report No. DESY-84-097, 1984, http:// cds.cern.ch/record/155607? $\ln =$ en.

[23] W. R. Faust, Transient behavior of a cavity, J. Appl. Phys. 43, 3983 (1972).

[24] CST Solvers, CST Solvers Website, also available as https://www.3ds.com/products-services/simulia/products/ cst-studio-suite/solvers/.

[25] E. Jensen, RF cavity design, Report No. CERN-2014-009, 2014, https://doi.org/10.5170/CERN-2014-009.405.

[26] J. D. Jackson, Classical Electrodynamics, 3rd ed. (Wiley, New York, NY, 1999) Chap. Waveguides, pp. 368-371, http://cdsweb.cern.ch/record/490457.

[27] S. Heifets and B. Zotter, On the symmetry of the impedance [particle accelerator], in Proceedings of the 1999 Particle Accelerator Conference (IEEE, New York, 1999), Vol. 2, pp. 1118-1119, https://doi.org/10.1109/PAC.1999 .795467.

[28] L. Palumbo, V. G. Vaccaro, and M. Zobov, Wake fields and impedance, Report No. CERN-95-06, CERN, Geneva, Switzerland, 1995 [arXiv:physics/0309023]. 